\title{
Transcriptional variation of sensory-related genes in natural populations of Aedes albopictus
}

Ludvik M. Gomulski ${ }^{1}$, Mosè Manni ${ }^{1,2}$, Davide Carraretto ${ }^{1}$, Tony Nolan ${ }^{3}$, Daniel Lawson ${ }^{4}$, José M. Ribeiro ${ }^{5}$, Anna R. Malacrida ${ }^{1}$ and Giuliano Gasperi ${ }^{1 *}$

\begin{abstract}
Background: The Asian tiger mosquito, Aedes albopictus, is a highly dangerous invasive vector of numerous medically important arboviruses including dengue, chikungunya and Zika. In four decades it has spread from tropical Southeast Asia to many parts of the world in both tropical and temperate climes. The rapid invasion process of this mosquito is supported by its high ecological and genetic plasticity across different life history traits. Our aim was to investigate whether wild populations, both native and adventive, also display transcriptional genetic variability for functions that may impact their biology, behaviour and ability to transmit arboviruses, such as sensory perception.

Results: Antennal transcriptome data were derived from mosquitoes from a native population from Ban Rai, Thailand and from three adventive Mediterranean populations: Athens, Greece and Arco and Trento from Italy. Clear inter-population differential transcriptional activity was observed in different gene categories related to sound perception, olfaction and viral infection. The greatest differences were detected between the native Thai and the Mediterranean populations. The two Italian populations were the most similar.

Nearly one million quality filtered SNP loci were identified.

Conclusion: The ability to express this great inter-population transcriptional variability highlights, at the functional level, the remarkable genetic flexibility of this mosquito species. We can hypothesize that the differential expression of genes, including those involved in sensory perception, in different populations may enable Ae. albopictus to exploit different environments and hosts, thus contributing to its status as a global vector of arboviruses of public health importance.

The large number of SNP loci present in these transcripts represents a useful addition to the arsenal of highresolution molecular markers and a resource that can be used to detect selective pressure and adaptive changes that may have occurred during the colonization process.
\end{abstract}

Keywords: Asian tiger mosquito, Invasive species, Differential transcription, Single nucleotide polymorphisms, Chemoreception

\footnotetext{
* Correspondence: gasperi@unipv.it

'Department of Biology and Biotechnology, University of Pavia, Pavia, Italy

Full list of author information is available at the end of the article
}

(c) The Author(s). 2020 Open Access This article is licensed under a Creative Commons Attribution 4.0 International License, which permits use, sharing, adaptation, distribution and reproduction in any medium or format, as long as you give appropriate credit to the original author(s) and the source, provide a link to the Creative Commons licence, and indicate if changes were made. The images or other third party material in this article are included in the article's Creative Commons licence, unless indicated otherwise in a credit line to the material. If material is not included in the article's Creative Commons licence and your intended use is not permitted by statutory regulation or exceeds the permitted use, you will need to obtain permission directly from the copyright holder. To view a copy of this licence, visit http://creativecommons.org/licenses/by/4.0/ The Creative Commons Public Domain Dedication waiver (http://creativecommons.org/publicdomain/zero/1.0/) applies to the data made available in this article, unless otherwise stated in a credit line to the data. 


\section{Background}

The Asian tiger mosquito, Aedes albopictus, is one of the most invasive species in the world and is increasingly becoming an important vector of virus-induced diseases (chikungunya, dengue, Zika) [1]. From its home-range in tropical Southeast Asia, where it was a zoophilic forest species [2], it spread initially to the Indian and Pacific islands [3] and then over the last 40 years, it rapidly spread to numerous tropical, subtropical and temperate regions in Europe, the Americas and Africa [4-7]. It is able to tolerate climate/environment interactions that differ from those of its home range [8-10]. Its ability to readily adapt to new habitats is associated with numerous environmentally-induced adaptive biological traits including eggs that can diapause and survive in cooler, less favourable conditions $[2,4,10,11]$ and opportunistic feeding behaviour on a wide range of animals [12]. Human activities also create new breeding and trophic niches of adaptation in the vicinity of dwellings, strengthening their association with humans [13, 14]. The threat of viral transmission has led to the establishment of surveillance and vector control strategies that aim to minimize the impact on public health. Successful intervention and control gains from knowledge of the genetic, ecological and behavioural traits of mosquito populations [15], as well as the history and dynamics of invasive processes. Numerous studies have attempted to unravel different aspects of the Ae. albopictus invasion process and to infer the genetic relationships between populations at the micro- and macro-geographic levels $[10,14,16-23]$. From these studies it appears that the global invasion process was aided by independent transand inter-continental introductions that may have facilitated the rapid establishment of adventive populations through admixture of unrelated genomes. As a result, a great deal of intra-population variability has been detected in both Southeast Asian populations and in the globally distributed adventive populations [19]. We have found that this genetic variability extends to the genetic mechanisms controlling vector competence for the chikungunya virus [19]. Here we used a comparative approach to verify whether this high genetic variability is also present at the transcriptional level.

We chose to sample the antennal transcriptomes of Ae. albopictus as the antennae are highly sophisticated peripheral sensory structures that act as intermediates between the mosquito, its environment and hosts. The antennae are implicated in the sensory reception of stimuli such as sound, heat, and odours [24] that are of vital importance to mosquitoes for mate location, the detection of suitable blood meal hosts, nectar sources, resting and oviposition sites [25]. Given the importance of the antennae in interactions of the mosquito with blood meal hosts, they will also influence disease transmission [26-28]. Moreover, the analysis of genetic diversity in sensory perception may help to improve the efficiency of control interventions [29, 30].

On this basis, we compared the antennal transcriptomes of wild Ae. albopictus samples collected from a population in the home range, Thailand, and populations from the Mediterranean invasion area with the aim of investigating the presence of transcriptional variation for genes controlling sensory perception functions. We extended our analyses to determine the presence and nature of single nucleotide polymorphisms (SNPs) in the transcripts present in the considered populations. We discovered the presence of a high level of interpopulation transcriptional and SNP variability in sensory related genes. High levels of variability were detected in genes associated with sound, vision and odorant perception, nitrogen assimilation, and flight behaviour. We also identified variability related to viral replication in the populations. The ability to express this extensive interpopulation genetic variability highlights the remarkable genetic flexibility of this mosquito species, that invaded much of the world in four decades, while its counterpart, Aedes aegypti, did so in four centuries [5].

\section{Methods \\ Mosquito samples}

Eggs were collected from three eco-geographic areas: one from the native range in Southeast Asia, Thailand, Ban Rai, Lampang Province $\left(18^{\circ} 18^{\prime} 36^{\prime \prime} \mathrm{N}\right.$ 99 $33^{\prime} 00^{\prime \prime} \mathrm{E}$, three collections, May-June 2012) and three from the Mediterranean area, Greece, Athens (37 $57^{\prime} 52^{\prime \prime} \mathrm{N} 23^{\circ} 43^{\prime}$ 44"E, one collection, September 2011) and northern Italy, Arco $\left(45^{\circ} 55^{\prime} 42^{\prime \prime} \mathrm{N} 10^{\circ} 56^{\prime} 02^{\prime \prime} \mathrm{E}\right.$, two collections,

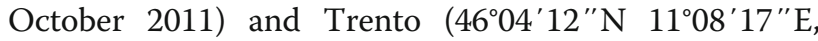
two collections, September 2011). The Ban Rai population derives from a tropical savanna climate (Köppen climate classification) with a mean summer temperature of around $35^{\circ} \mathrm{C}$. Monthly rainfall during the collection period averaged $140 \mathrm{~mm}$. The area is a mixture of woodland and agricultural land with mainly rice cultivation. The Athens population derives from a temperate, highly urbanised setting with high levels of anthropogenic environmental transformation (anthropization) and pollution [31]. The average temperature during the collection period was $25^{\circ} \mathrm{C}$ with a monthly precipitation of $23 \mathrm{~mm}$. The Trentino/Arco populations are from a mostly mountainous and forested area with a temperate climate, and a lower extent of human intervention with limited agricultural use. Average monthly temperature during the collection period was $19.9^{\circ} \mathrm{C}$ and $91 \mathrm{~mm}$ rainfall for Trento and $13.4^{\circ} \mathrm{C}$ and $100 \mathrm{~mm}$ rainfall for Arco. For each locality, the eggs were collected and pooled from several oviposition traps to minimize inbreeding effects. The eggs were brought into the insectary maintained at 
$26^{\circ} \mathrm{C}$ with $70 \%$ relative humidity and a $12: 12 \mathrm{~h}$ (light: dark) photoperiod and were hatched in autoclaved water and fed on fish food pellets (Tetra) daily as necessary. Pupae were collected in plastic cups containing $50 \mathrm{ml}$ water and placed in $20 \mathrm{~cm}$ cube cages from which newly emerged adults were collected each morning. Both sexes were maintained together in the same cage with 20\% sugar solution at the same temperature/humidity/photoperiod as previously described. At 2 to 3 days of age the antennae were removed from equal numbers of G0 male and female individuals from each population sample and placed immediately in $200 \mu \mathrm{l}$ of chilled Trizol (Invitrogen). The number of antennae pairs collected per population was 420 for Arco, 435 for Athens, 764 for Ban Rai and 921 for Trento.

\section{RNA sample preparation and sequencing}

For each population collection, total RNA was extracted from the antennae using Trizol (Invitrogen), followed by treatment with DNase (DNAfree, Ambion). The RNA sample from each collection was further purified using Micro Bio-Spin P-30 gel columns (BioRad). After quantification using an Agilent 2100 Bioanalyser the RNAs from the different collections in each of the four sampling sides were pooled. mRNA isolation and cDNA library preparation for each of the four samples were performed using the Illumina TruSeq RNA sample preparation kit (Illumina Inc., San Diego, CA, USA). The libraries were barcoded, pooled and sequenced as a 100 bp paired end run on one lane of an Illumina HiSeq 2000 platform at a concentration of $8 \mathrm{pM}$.

\section{Bioinformatics analyses}

The paired-end sequences from the four population samples were combined, stripped of terminal primers using a local script based on the NCBI Vector Screen tool [32] and assembled with ABySS [33] and Soapdenovo-Trans [34] using k parameters from 25 to 95 at intervals of 5, and Trinity [35]. The assemblies were joined using a iterative blast and CAP3 pipeline as previously described [36]. The results of these analyses were then piped into a hyperlinked Excel report, as described in the dCAS software tool [37]. The coding sequences (CDS) that encoded putative polypeptides of at least 40 amino acids (aa) were extracted according to i) matches to proteins in the NCBI nr database; ii) the largest ORF. Functional annotations of the transcripts were performed using the program Classifier (Ribeiro, unpublished), which combines the output of several tools: BLASTX to compare the nucleotide sequences to the NCBI nr protein database, Swissprot; rpsblast to search for conserved protein domains in Pfam, SMART, KOG, Conserved Domains Databases (CDD) and GO databases. Transcripts were also compared to mitochondrial and rRNA nucleotide sequences from NCBI.

Bowtie2 version 2.1.0 was used to index the complete assembled transcriptome derived from the pooled reads from the four populations. RNA-seq paired-end reads for each population were mapped independently using TopHat2 2.0.9 [38] against the combined transcriptome contigs with default parameters except for a maximum number of 3 mismatches. The resulting BAM format alignments were used as input for Cufflinks 2.1.1 [39]. Significance tests for differential transcript abundances were determined using Cuffdiff with a false discovery rate (FDR) of 0.05 . The intersections among the four population samples (i.e. more abundant transcripts in one or more samples in comparisons with the other samples) of the antennal genes were visualized by a Venn diagram [40]. The Cuffdiff results were also visualized and explored using CummeRbund v. 2.0.0 [41] and additional statistical analyses were performed using $\mathrm{R}$ version 3.6.2 [42]

Gene ontology analysis was performed with Blast2GO [43]. Over- and under-representation of multiple-level GO categories [44] within the over-abundant transcripts identified by the Cuffdiff pair-wise analyses was evaluated using the Fisher's Exact Test within Blast2GO (Enrichment analysis) with a false discovery rate (FDR) of 0.05 .

To determine the phylogenetic relationships of odorant binding protein (OBP) and odorant receptor (OR) sequences encoding at least 100 or 150 amino acids, respectively, with their Ae. aegypti counterparts, the amino acid sequences (excluding signal peptide sequences, where present) were aligned using MAFFT v7 [45] with the E-INS-i strategy, BLOSUM62 matrix, 1000 maxiterate and offset 0 . Phylogenetic relationships were estimated using Maximum Likelihood with 1000 bootstrap replications with MEGA 6.0.6 [46] and mid-point rooted trees were plotted using FigTree v1.4 [47].

Transmembrane domains in identified odorant receptor protein sequences were predicted using MEMSAT3 [48] and snake plots of the predicted structures were generated using TOPO2 [49].

SNP analyses were performed in accordance with the GATK best practices for variant calling on RNAseq [50]. The TopHat2 BAM files were sorted with respect to the de-novo reference transcriptome using the ReorderSam tool in Picard 1.108 [51], read groups were added, duplicates marked, and indexes were created using the Picard tools and reassignment of mapping qualities was performed using SplitNCigarReads tool in GenomeAnalysisTK (GATK 3.0-0) [52]. Variant calling was performed simultaneously for the four population libraries using the GATK HaplotypeCaller tool with a minimum phredscaled threshold of 20. Variant filtering was performed 
using the GATK VariantFiltration tool to screen Fisher Strand values (FS $>30)$, quality by depth values $(\mathrm{QD}<$ 2.0) and clusters of at least 3 SNPs within a window of 35 bases. The Fisher Strand values are an assessment of strand bias (the variation being seen on only the forward or only the reverse strand) where more bias is indicative of false positive calls. The predicted effects of the SNP variants (synonymous/nonsynonymous) on the putative amino acid product were determined manually using CLC Main Workbench 6 (Qiagen) and MEGA 6.0.6. The numbers of fixed or polymorphic non-synonymous/ synonymous sites relative to Aedes aegypti were used to assess the selective pressures acting on genes using the McDonald-Kreitman test [53] using DnaSP 6.12 .03 [54] considering sites with a minimum coverage of 10 reads in the sorted, indexed BAM files as determined using Integrative Genomics Viewer (IGV) 2.5.3 [55]. Additional statistical analyses were performed using $\mathrm{R}$ version 3.6.2 [42].

\section{Reverse transcriptase-PCR (RT-PCR) for the analysis of the tissue-specificity of OBP/OR transcripts}

Total RNA was extracted using Trizol (Invitrogen) from different body compartments of 2-4 day old male and female Ae. albopictus that had been maintained together in the same cage since emergence. Pools of each of the following were used: antennae ( $\sim 100$ pairs $)$, palps $(\sim 100$ pairs), proboscises (25), heads without antennae, palps and proboscises (5), tarsi ( 10 sets), legs without tarsi $(\sim 10$ sets), thoraces without wings and legs (5), abdomens (5) and wings (50 pairs). After DNAse treatment (DNAfree, Ambion), RNA integrity was determined by formaldehyde agarose gel electrophoresis and quantified using a Nanodrop ND-1000 spectrophotometer (Nanodrop Technologies). For each body part $200 \mathrm{ng}$ of the extracted total RNA was transcribed into cDNA using the iScript $^{\text {tw }}$ cDNA Synthesis Kit (Biorad). RT-PCRs with gene specific primers, designed using Primer3Plus version 2.3.6 [56](Additional file 1: Table S1) were performed using $5 \%$ of the synthesized cDNA and the following cycle conditions: $94^{\circ} \mathrm{C}$ for $3 \mathrm{~min}, 30$ cycles at $94{ }^{\circ} \mathrm{C}$ for $30 \mathrm{~s}, 58^{\circ} \mathrm{C}$ for $30 \mathrm{~s}, 72^{\circ} \mathrm{C}$ for $2 \mathrm{~min}$, and a final extension at $72{ }^{\circ} \mathrm{C}$ for $10 \mathrm{~min}$. The Ae. albopictus RpL34 gene (GenBank accession no. AF144549) was amplified as a control for cDNA integrity. To control for genomic DNA contamination, RT-PCR was also performed on samples in which cDNA synthesis had been performed in the absence of reverse transcriptase. The amplification products were electrophoresed on $2 \%$ agarose gels.

\section{Validation of RNAseq with real-time quantitative PCR} Real-time quantitative PCR (qRT-PCR) was performed on six genes (Aalb-no mechanoreceptor potential $C$ (nompC), a carboxylesterase (Aalb-CCEae3a), a cytochrome P450 (Aalb-cyp450) and three odorant binding proteins, AalbOBP17 (Aalb-6031), AalbOBP62 (Aalb-88,196) and AalbOBP75 (Aalb-4806) using cDNA derived from the antennae of 2 to 3 day-old male and female individuals from lines derived from Trento $\left(\mathrm{F}_{4}\right)$, Athens $\left(\mathrm{F}_{3}\right)$ and Ban Rai $\left(\mathrm{F}_{2}\right)$. Two reference genes, Aalb-G6PDH (Aalb-91,038) and Aalb-RpL34 (GenBank accession no. AF144549) were used for relative quantification normalization [57] (Additional file 2: Table S2). $R p L 34$ has been used as a reference gene in this species in several previous studies $[58,59]$. Synthesis of cDNA was performed using $100 \mathrm{ng}$ RNA in $20 \mu \mathrm{l}$ reaction volumes using the iScript cDNA Synthesis kit (Bio-Rad). Real-time quantification was performed using the SsoFast EvaGreen Supermix kit (Bio-Rad) and MasterCycler realplex (Eppendorf). Cycling conditions involved an initial $95^{\circ} \mathrm{C}$ for $30 \mathrm{~s}, 40$ cycles of $10 \mathrm{~s}$ at $95^{\circ} \mathrm{C}, 30 \mathrm{~s}$ at $58^{\circ} \mathrm{C}$. A fluorescence reading was made at the end of each extension step. Three biological replicates were performed, and the specificity of the amplification products was assessed by melt-curve analysis. Statistical comparison of RNAseq and qRT-PCR (log2 ratio) datasets was performed using Pearson correlation analysis [42].

\section{Results \\ Differential transcription in the antennae of mosquitoes from different geographic populations}

Over 420 million reads were generated from the antennae of male and female mosquitoes from the native Thai (Ban Rai, 140.6 M) and three adventive Mediterranean populations, Greece (Athens, $105.9 \mathrm{M}$ ) and Italy (Arco, 64.6 $\mathrm{M}$ and Trento, 109.0 M). The reads were assembled into 98,534 contigs with an N50 of $1296 \mathrm{bp}$. BLASTN of these contigs against both the Fellini and Foshan genomes $[60,61]$ resulted in hit rates of 95\% (4464 and 4999 contigs, respectively, gave no hits with an expectation, $e<10^{-6}$ ). When queried against the protein $\mathrm{nr}$ database and categorized into different gene ontology (GO) functional classes, the most frequent level III terms were ion binding, organic cyclic compound binding, heterocyclic compound binding, protein binding and small molecule binding (Fig. 1).

The four population samples, based on transcription levels (FPKM), were highly significantly different (paired Wilcoxon-Pratt signed rank test with continuity correction, $P<0.0001)$. This is evident also in a Principal Component Analysis (PCA, prcomp package, R) where component 1 (accounting for $47.8 \%$ of the variation), separates the native Thai population from the other three adventive populations, especially the two Italian populations. The second component (accounting for $31.5 \%$ of the variation) effectively separates the Athens population from the two Italian populations (Fig. 2). This differentiation of the populations was evident as 


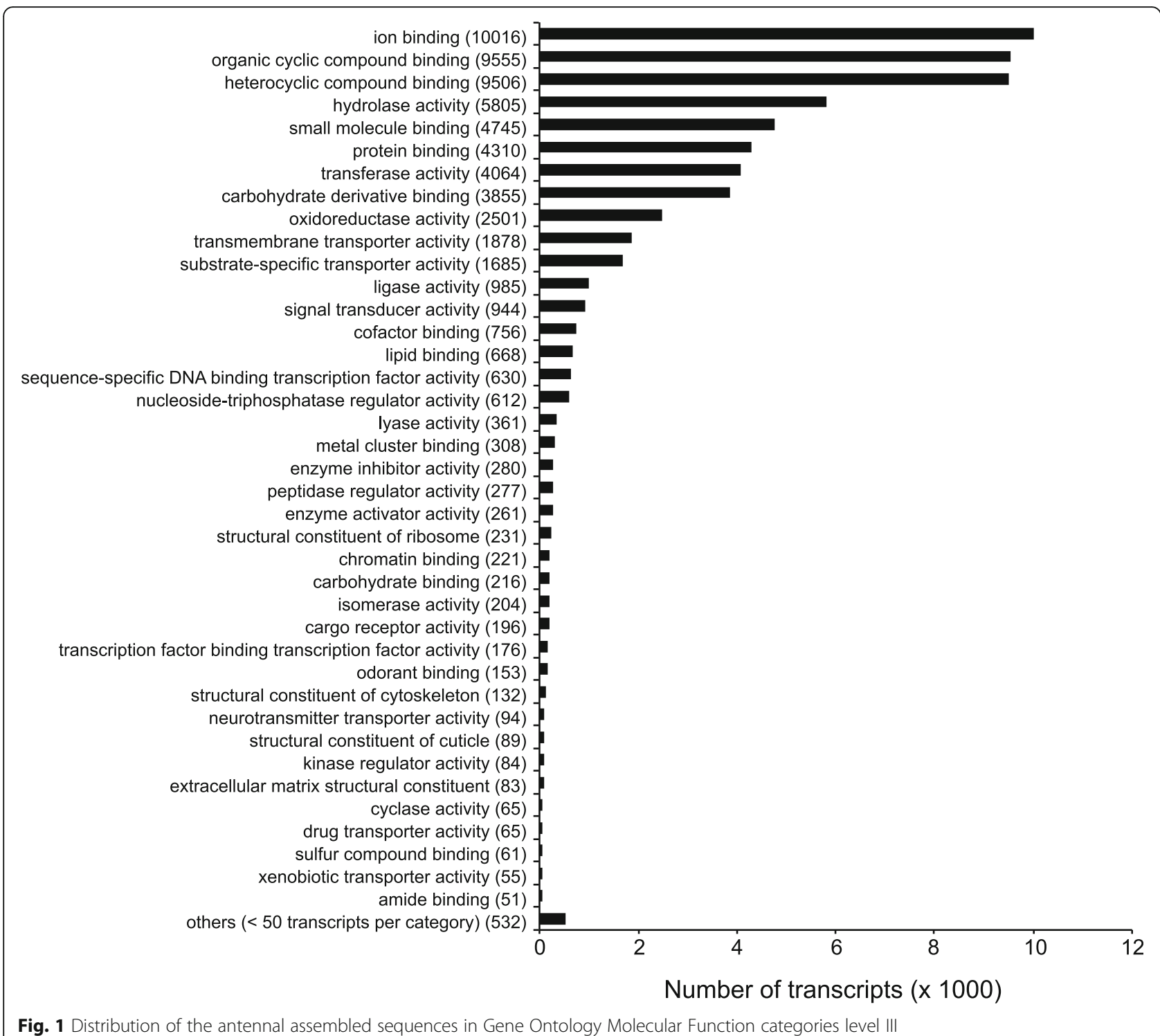

7418 of the transcripts displayed differential abundances in the four population samples with a False Detection Rate (FDR) of 0.05. These differentially abundant transcripts belong to a wide range of GO terms, (Additional file 3: Figure S1) including ion binding, organic cyclic binding, protein binding and odorant binding terms.

Significantly more abundant transcripts in the different population samples and those shared between the different samples are shown in the Venn diagram (Fig. 3a). The Athens and the Ban Rai populations have the highest number of unique more abundant transcripts (2063 and 2210, respectively) and share a total of 553 more abundant transcripts of which 515 are shared only by them. Lower numbers of unique more abundant transcripts were present in the two Italian Arco and Trento populations (794 and 642, respectively) and they alone share 561 transcripts.

The number of transcripts with differential abundances varied greatly in the different population pairwise comparisons (Fig. 3b). The comparison between the native Thai (Ban Rai) sample and Arco resulted in the most differential abundances (3543 transcripts), with $37 \%$ more abundant in Arco and 63\% in Ban Rai. Whereas, the two geographically related north Italian populations, Arco and Trento, showed the lowest number of differential abundances (387 transcripts), 26\% of which were more abundant in Arco and 74\% in Trento.

The nature of the antennal transcriptional differences between the population samples was explored by Enrichment analyses. When compared to the complete dataset there was significant over- and under-representation of 


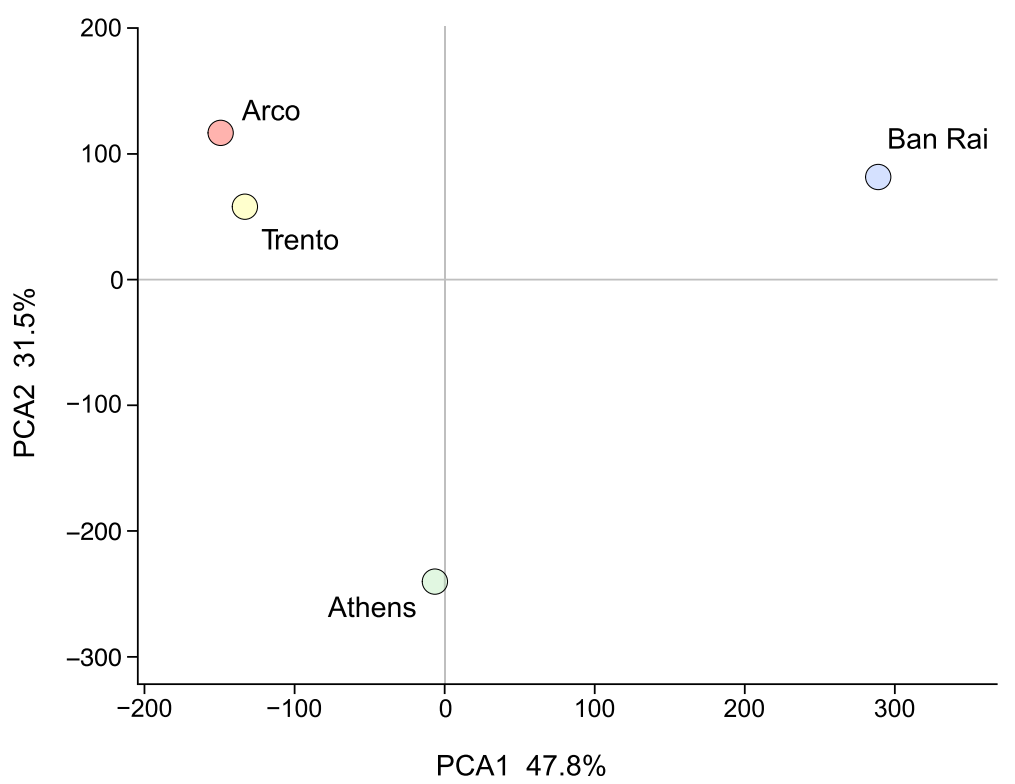

Fig. 2 Principal Component Analysis based on transcript abundance illustrating the relationships between the four population samples

GO categories among the differentially abundant transcripts present in many pairwise comparisons of the samples. The terms that were enriched in the different comparisons are summarised in Table 1. Full details of the terms are given in Additional file 4: Tables S3-S8.

As a confirmation of the validity of the RNA-seq transcript abundance estimates, a highly significant positive correlation $(r=0.9119, P<0.0001)$ was found between the RNAseq and qRT-PCR datasets derived from the abundance of six transcripts in three population samples, Trento, Athens and Ban Rai (Additional file 5: Figure S2, Additional file 6: Table S9).

\section{Functional categories of antennal transcripts that are differentially abundant in the four populations Sensory perception of smell}

Functional categories related to odorant perception and sensory perception of smell were found to be overrepresented in the pairwise population antennal comparisons. Out of a total of 162 transcripts with significant similarity to odorant binding proteins (OBPs), 32 were identified as members of the Classic subfamily and 12 of the Plus-C subfamily [62] (Table 2, Additional file 7: Figure S3, Additional file 8: Table S10). No members of the Two-domain subfamily were identified. A repertoire of 242 transcripts shared significant sequence similarity with known odorant receptor (OR) proteins $\left(\mathrm{e}<10^{-6}\right)$ from other species, and particularly from Ae. aegypti [63, 64]. Out of the considered 80 transcripts (Table 3), 49 encoded complete ORs ranging in size from 375 to 479 amino acids, whereas two appeared to be pseudogenes
(Aalb-5361 and Aalb-81,229) as, despite being full length, they contained an internal stop codon or a frame-shift, respectively (Additional file 9: Figure S4, Additional file 8: Table S11).

Seven contigs, encoding polypeptides between 53 to 273 amino acids in length, shared significant sequence similarity with known gustatory receptor (GR) proteins (Additional file 10: Table S12).

Within the OBP functional category three OBP transcripts were found to be differentially transcribed across the four population samples; the Classic subfamily Aalb6031/AalbOBP17 member and two Plus-C subfamily members, Aalb-4806/AalbOBP75 and Aalb-88,196/AalbOBP62. The Classic AalbOBP17 was enriched 4.2-fold in Arco with respect to Athens $(P=0.0047)$ and 3 -fold compared to Ban Rai $(P=0.0336)$. While the Plus-C AalbOBP75 was enriched 14-fold in Arco compared to both Athens and Ban Rai $(P=0.0389$ and $P=0.0373$, respectively). The other Plus-C OBP, AalbOBP62 was enriched 2.9 -fold in Arco compared to Athens $(P=0.0189)$. The relative abundances of the OBP transcripts varied greatly and ranged from a mean FPKM value of 4.1 for Aalb-57, $651 /$ AalbOBP73 to a mean FPKM value of 19,539 for Aalb-96,031/AalbOBP3 (Fig. 4). Also, in terms of tissue specificity the three differentially abundant OBPs are heterogeneous. The Plus-C AalbOBP75 appeared to be specific for the antennae of males and females. The other Plus-C AalbOBP62 displays a wider tissue distribution also in relation to sexes; it is transcribed in the antennae, head and tarsi of both sexes but also in the female maxillary palps and proboscis. The Classic 
A

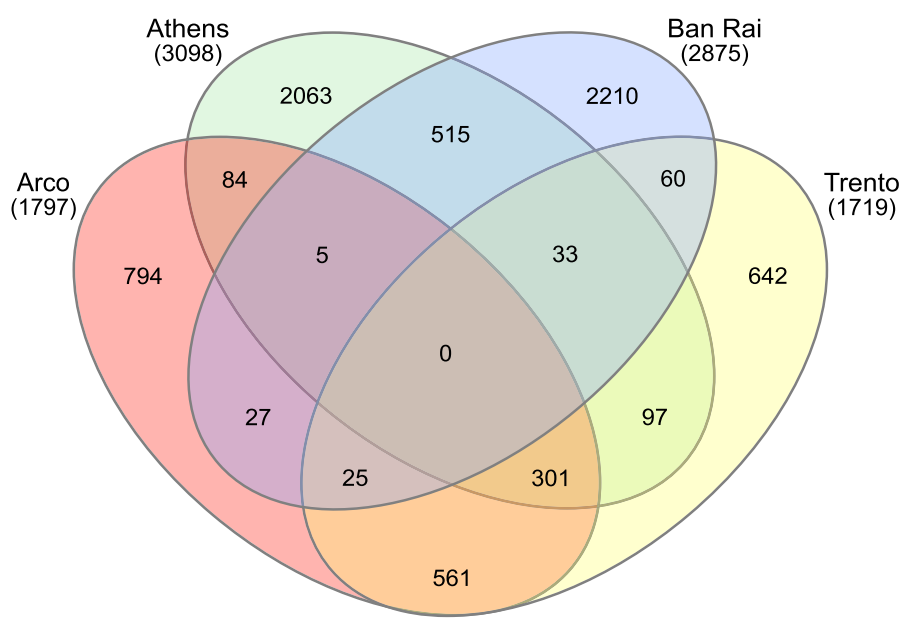

B

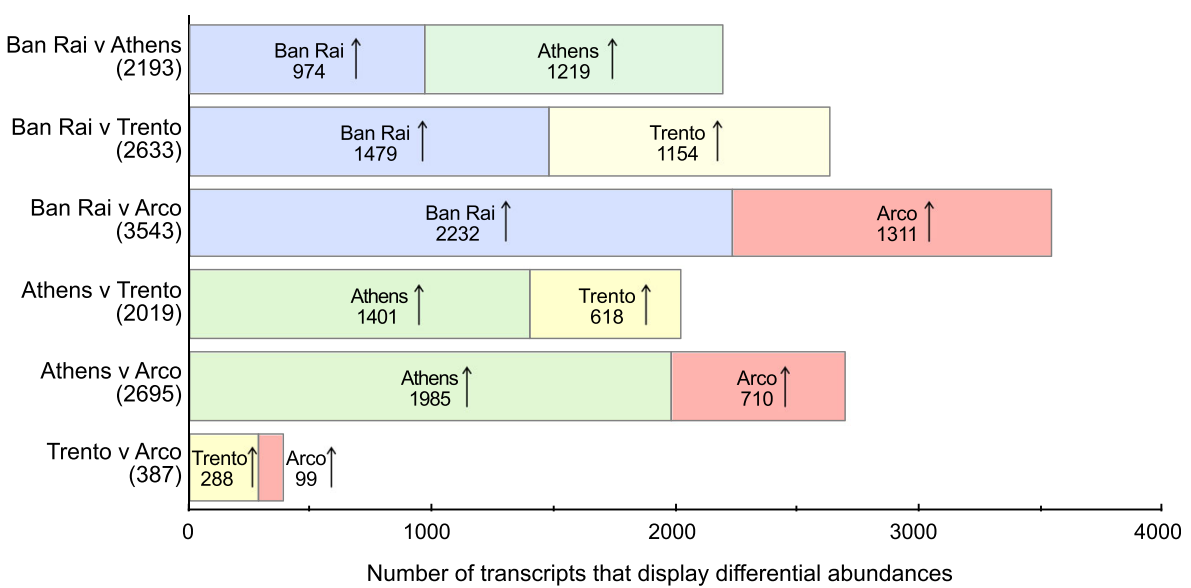

Fig. 3 Differential abundances of transcripts in the different population samples. a Venn diagram illustrating the number of more abundant transcripts shared in the different population samples. $\mathbf{b}$ Numbers of transcripts that displayed differential abundances in the pairwise comparisons between the population samples. Arrows indicate that transcript abundance was greater in the respective population sample

Table 1 Enriched Gene Ontology categories in the different Ae. albopictus population sample comparisons

\begin{tabular}{lll}
\hline Comparison & Population & Summary of enriched GO categories \\
\hline Arco $\vee$ Trento & Arco & Regulation and maintenance of photoreception \\
& Trento & - \\
Athens $\vee$ Trento & Athens & Nitrogen assimilation; oxidative processes \\
& Trento & Viral attachment and entry, viral genome replication \\
Athens $\vee$ Ban Rai & Athens & Iron-ion binding \\
& Ban Rai & - \\
Ban Rai $\vee$ Trento & Ban Rai & Nitrogen assimilation; oxidative processes \\
& Trento & Viral attachment and entry, viral genome replication \\
Arco $\vee$ Athens & Arco & Viral attachment and entry, viral genome replication; odorant binding; photo transduction \\
& Athens & Nitrogen assimilation; oxidative processes; perception of sound \\
Arco $\vee$ Ban Rai & Arco & Viral attachment and entry, viral genome replication; odorant binding; photo transduction; oxidative processes \\
& Ban Rai & Perception of sound, heat and smell; flight behaviour \\
\hline
\end{tabular}


Table 2 Aedes albopictus putative odorant binding protein (OBP) transcripts

\begin{tabular}{|c|c|c|c|c|c|c|c|c|}
\hline \multirow{3}{*}{$\begin{array}{l}\text { Transcript } \\
\text { Aalb-88,094 }\end{array}$} & \multirow{3}{*}{$\begin{array}{l}\text { aa } \\
143\end{array}$} & \multirow{3}{*}{$\begin{array}{l}\text { Name } \\
\text { AalbOBP1 }\end{array}$} & \multicolumn{2}{|c|}{ Ae. aegypti orthologue } & \multicolumn{4}{|c|}{ Signal Peptide } \\
\hline & & & Gene & Name & Pos, D & Subfamily & & Complete $^{a}$ \\
\hline & & & AAEL009449 & AaegOBP1 & $18,0.735$ & Classic & OS-E/OS-F & \\
\hline Aalb-89,777 & 159 & AalbOBP2 & AAEL006176 & AaegOBP2 & $24,0.752$ & Classic & Pbprp1 & \\
\hline Aalb-96,031 & 143 & AalbOBP3 & AAEL013018 & AaegOBP3 & $18,0.747$ & Classic & OS-E/OS-F & \\
\hline Aalb-88,146 & 134 & AalbOBP4 & AAEL000073 & AaegOBP4 & $26,0.747$ & Classic & OBP19a & \\
\hline Aalb-4635 & 132 & AalbOBP9 & AAEL002596 & AaegOBP9 & $20,0.643$ & Classic & mclassic3 & \\
\hline Aalb-90,263 & 140 & AalbOBP10 & AAEL007603 & AaegOBP10 & $25,0.890$ & Classic & mclassic6 & \\
\hline Aalb-88,450 & 137 & AalbOBP11 & AAEL002587 & AaegOBP11 & $18,0.901$ & Classic & mclassic3 & \\
\hline Aalb-97,431 & 132 & AalbOBP12 & AAEL002617 & AaegOBP12 & $18,0.850$ & Classic & mclassic3 & \\
\hline Aalb-88,277 & 133 & AalbOBP13 & AAEL002591 & AaegOBP13 & $18,0.873$ & Classic & mclassic4 & \\
\hline Aalb-25,065 & 136 & AalbOBP15 & AAEL002598 & AaegOBP15 & $23,0.833$ & Classic & mclassic1 & \\
\hline Aalb-6031 & 138 & AalbOBP17 & AAEL004339 & AaegOBP17 & $19,0.813$ & Classic & mclassic7 & \\
\hline Aalb-98,240 & 141 & AalbOBP18 & AAEL004342 & AaegOBP18 & $22,0.927$ & Classic & mclassic9a & \\
\hline Aalb-1010 & 125 & AalbOBP19 & AAEL004343 & AaegOBP19 & - & Classic & mclassic9a & $\mathrm{N}$ \\
\hline Aalb-51,548 & 142 & AalbOBP19-N1 & AAEL004343 & AaegOBP19 & $20,0.902$ & Classic? & - & \\
\hline Aalb-88,295 & 166 & AalbOBP20/59 & AAEL005778 & AaegOBP20 & $24,0.762$ & Classic & Pbprp4 & \\
\hline Aalb-2021 & 138 & AalbOBP22 & AAEL005772 & AaegOBP22 & $16,0.775$ & Classic & Obp99a & \\
\hline Aalb-57,665 & 101 & AalbOBP26/23 & AAEL006109 & AaegOBP23 & - & Plus-C & mplus11 & $\mathrm{N}, \mathrm{C}$ \\
\hline Aalb-10,170 & 200 & AalbOBP25/24 & AAEL006103 & AaegOBP25 & $20,0.796$ & Plus-C & mplus11 & \\
\hline Aalb-95,920 & 139 & AalbOBP27 & AAEL000071 & AaegOBP27 & $19,0.903$ & Classic & OBP19a & \\
\hline Aalb-88,172 & 149 & AalbOBP34 & AAEL014082 & AaegOBP34 & $24,0.865$ & Classic & LUSH & \\
\hline Aalb-86,836 & 131 & AalbOBP35 & AAEL002606 & AaegOBP35 & $18,0.904$ & Classic & mclassic3 & \\
\hline Aalb-91,258 & 152 & AalbOBP36 & AAEL008011 & AaegOBP36 & $20,0.795$ & Classic & OS-E/OS-F & \\
\hline Aalb-94,240 & 149 & AalbOBP37 & AAEL008009 & AaegOBP37 & $20,0.940$ & Classic & OS-E/OS-F & \\
\hline Aalb-90,197 & 140 & AalbOBP38 & AAEL008013 & AaegOBP38 & $16,0.883$ & Classic & OS-E/OS-F & \\
\hline Aalb-91,445 & 146 & AalbOBP39 & AAEL006454 & AaegOBP39 & $21,0.719$ & Classic & LUSH & \\
\hline Aalb-88,422 & 157 & AalbOBP63/42 & AAEL010666 & AaegOBP42 & No & Plus-C & mplus1 & \\
\hline Aalb-88,397 & 191 & AalbOBP47 & AAEL011499 & AaegOBP47 & $20,0.909$ & Plus-C & mplus1 & \\
\hline Aalb-87,394 & 151 & AalbOBP55 & AAEL012377 & AaegOBP55 & $21,0.690$ & Classic & OBP19a & \\
\hline Aalb-88,160 & 140 & AalbOBP56 & AAEL000051 & AaegOBP56 & $19,0.601$ & Classic & OBP19a & \\
\hline Aalb-92,750 & 155 & AalbOBP59-N1 & AAEL015313 & AaegOBP59 & $21,0.812$ & Classic & Pbprp4 & \\
\hline Aalb-88,453 & 122 & AalbOBP60 & AAEL015499 & AaegOBP60 & $18,0.877$ & Classic & OS-E/OS-F & $C$ \\
\hline Aalb-88,196 & 193 & AalbOBP62 & AAEL015566 & AaegOBP62 & $21,0.764$ & Plus-C & mplus1 & \\
\hline Aalb-89,129 & 181 & AalbOBP69 & AAEL000124 & AaegOBP69 & $24,0.816$ & Plus-C & mplus7 & \\
\hline Aalb-74,008 & 156 & AalbOBP72 & AAEL004729 & AaegOBP72 & - & Plus-C & mplus8 & $\mathrm{N}$ \\
\hline Aalb-57,651 & 166 & AalbOBP73 & AAEL004730 & AaegOBP73 & $21,0.780$ & Plus-C & mplus8 & $\mathrm{N}$ \\
\hline Aalb-45,154 & 169 & AalbOBP73-N1 & AAEL004730 & AaegOBP73 & $23,0.813$ & Plus-C & mplus8 & \\
\hline Aalb-4806 & 193 & AalbOBP75 & AAEL011483 & AaegOBP75 & $19,0.748$ & Plus-C & mplus5 & \\
\hline Aalb-96,930 & 133 & AalbOBP76 & AAEL007604 & AaegOBP76 & $17,0.765$ & Classic & mclassic6 & \\
\hline Aalb-46,119 & 120 & AalbOBP77 & AAEL002626 & AaegOBP77 & - & Classic & mclassic2 & $\mathrm{N}, \mathrm{C}$ \\
\hline Aalb-13,742 & 116 & AalbOBP78-N1 & AAEL001836 & AaegOBP78 & - & Classic & Bombyx mori & N \\
\hline Aalb-45,987 & 151 & AalbOBP81 & AAEL011730 & AaegOBP81 & $23,0.747$ & Classic & mclassic8 & \\
\hline
\end{tabular}


Table 2 Aedes albopictus putative odorant binding protein (OBP) transcripts (Continued)

\begin{tabular}{|c|c|c|c|c|c|c|c|c|}
\hline & & & Ae. aegypti or & & Signal Pep & & & \\
\hline Aalb-92,539 & 306 & AalbOBP83 & AAEL011416 & AaegOBP83 & $29,0.925$ & Classic & - & \\
\hline Aalb-23,731 & 197 & AalbOBP-N1 & - & - & $27,0.679$ & Plus-C & $?$ & \\
\hline Aalb-17,964 & 103 & AalbOBP-N2 & AAEL004721 & hypothetical protein & - & Plus-C & mplus4 & $\mathrm{N}$ \\
\hline
\end{tabular}

${ }^{a} \mathrm{~N}, \mathrm{C}$ : incomplete at $\mathrm{N}$ - and/or C-terminus

AalbOBP17 appeared to be transcribed in all body compartments, in both sexes, although the strongest signals were from the antennae (Fig. 5).

Within the OR functional category, two differentially abundant OR transcripts were identified across the four populations; Aalb-96,847/AalbOR100 was enriched 6 fold in Arco with respect to Ban Rai $(P=0.0174)$ while Aalb-97,870/AalbOR47-N2 was enriched 23 fold in Arco and 18 fold in Trento compared to Ban Rai $(P=0.0247$ and $P=0.0247$, respectively) (Fig. 6). The abundances of the OR transcripts differed enormously. The highest abundance (mean 868 FPKM in the four populations) was for AalbOR7/ORCO (Aalb-88,204), expected given its obligate co-expression with the ligand-specific tuning ORs. The other considered OR transcript abundances ranged from a mean FPKM value of 1.9 (Aalb-6097/AalbOR76) to 76.5 (Aalb-95,352/AalbOR11).

\section{Viral infection terms}

The over-representation of viral infection terms in the antennae of the north Italian populations, Arco and Trento compared to Athens and Ban Rai involved nine up-regulated sequences: Aalb-11,736, Aalb-24,050, Aalb24,228, Aalb-50,498, Aalb-52,817, Aalb-67,423, Aalb-71, 556, Aalb-77,037 and Aalb-82,640 which display very high identities (99-100\%) with parts of the polyprotein gene of Aedes flavivirus (AeFV) (GenBank acc. KC181923 [66] GenBank acc. AB488408 [67]). These AeFV transcripts were abundant in the two Italian populations (average FPKM = 6.7 and 9.7 in Arco and Trento, respectively) and practically absent in the Athens $(\mathrm{FPKM}=0.005) \quad$ and $\quad$ Ban $\quad$ Rai $\quad($ FPKM $=0.103)$ populations.

\section{Iron-ion binding terms}

Up-regulation of 14 cytochrome P450 transcripts, glutamate synthase, a carboxy/choline esterase, ABC transporter, aconitase and transferrin was found in Athens with respect to Trento and Thailand. Aalb-89,068, the homologue of AAEL005112, a Carboxy/cholinesterase alpha esterase, CCEae3a, showed up to 44-fold upregulation in Athens (119.4 FPKM) compared to the other population samples (5.4 FPKM in Arco, 2.7 in Ban Rai and 4.1 in Trento).

\section{Sound and visual perception terms}

Transcripts associated with the perception of sound were enriched in Athens and Ban Rai in the comparisons with Arco. These include several dynein intermediate heavy chain homologues and a nompC (no mechanoreceptor potential C) homologue. The nompC homologue (Aalb-91,878) was significantly up-regulated (4.2 fold) in Ban Rai compared to Arco (17.6 versus 4.2 FPKM, respectively). The enriched regulation and maintenance of photoreception GO category in Arco in the comparisons with Trento and Athens involved three transcripts (Aalb-90,651, Aalb-94,114, Aalb-93,715) of two arrestins (homologues of arrestin-1 and arrestin-2 in An. gambiae).

\section{Single nucleotide polymorphism across the antennal transcriptomes}

A total of 958,216 GATK quality filtered SNP loci were identified in the assembled sequences, however almost $54 \%$ of these loci were clustered (three or more SNPs within a window of 35 bases) and when these were discarded the number of SNP loci fell to 441,230. The mean SNP densities (sites with at least one alternative allele/kb) were significantly different in the population samples (Ban Rai 10.35; Athens 9.08; Trento 9.17 and Arco 7.30, $P=0.0$, bootstrap heteroscedastic one-way ANOVA for trimmed means (tiwaybt) followed by robust post hoc tests as implemented in the $\mathrm{R}$ package WRS2 [68]). The post hoc tests showed that the mean densities of the polymorphic sites were highly significantly different $(P<0.0001)$ in all but the Trento/ Athens pairwise comparison $(P=0.2304)$. The polymorphic site densities were not correlated with read depth (FPKM) in the population samples (Pearson's product-moment correlation: Arco, $r=0.0053, P=$ 0.237; Athens, $r=-0.0019, P=0.662$; Ban Rai, $r=$ $0.0068, P=0.1192$; Trento, $r=0.0056, P=0.2039$ ) indicating that read coverage did not bias SNP detection (Additional file 11: Figure S5). A PCA (prcomp package, R) (Fig. 7) based on the allelic frequencies of 254,336 biallelic SNP loci (with a filtered read depth of at least 20 in each population) was congruent with that derived from transcript levels (Fig. 2) with the first component (accounting for $59.8 \%$ of the variation), grouping the Athens and native Ban Rai populations and effectively 
Table 3 Aedes albopictus putative odorant receptor (OR) transcripts

\begin{tabular}{|c|c|c|c|c|c|c|c|}
\hline & & & Ae. aegypti o & & Conserve & & \\
\hline Transcript & aa & & Gene & Name $^{a}$ & Domain & e-value & Incomplete \\
\hline Aalb-16,280 & 376 & AalbOR2 & AAEL005999 & OR2 & $7 \mathrm{tm} \_6$ & 7.27E-47 & \\
\hline Aalb-5943 & 406 & AalbOR4/5 & AAEL015147 & OR4 & $7 \mathrm{tm} \_6$ & $9.72 \mathrm{E}-43$ & \\
\hline Aalb-82,460 & 401 & AalbOR6 & AAEL017548 & OR6 & $7 \mathrm{tm} \_6$ & $8.39 \mathrm{E}-37$ & \\
\hline Aalb-88,204 & 479 & AalbOR7/ORCO & AAEL005776 & OR7/ORCO & $7 \mathrm{tm} \_6$ & $5.66 \mathrm{E}-41$ & \\
\hline Aalb-8503 & 375 & AalbOR10 & AAEL006003 & OR10 & $7 \mathrm{tm} \_6$ & $3.27 \mathrm{E}-43$ & \\
\hline Aalb-95,352 & 420 & AalbOR11 & AAEL011583 & OR11 & $7 \mathrm{tm} \_6$ & 8.93E-31 & \\
\hline Aalb-83,056 & 389 & AalbOR13 & AAEL008368 & OR13 & $7 \mathrm{tm} \_6$ & $9.68 \mathrm{E}-28$ & \\
\hline Aalb-9257 & 390 & AalbOR15 & AAEL008448 & OR15 & $7 \mathrm{tm} \_6$ & 4.23E-18 & \\
\hline Aalb-92,214 & 400 & AalbOR18/19 & AAEL017017 & OR19 & $7 \mathrm{tm} \_6$ & $2.49 \mathrm{E}-13$ & \\
\hline Aalb-84,246 & 396 & AalbOR20 & AAEL004966 & OR20 & $7 \mathrm{tm} \_6$ & $5.72 \mathrm{E}-12$ & \\
\hline Aalb-47,088 & 396 & AalbOR21 & AAEL017398 & OR21 & $7 \mathrm{tm} \_6$ & $1.05 \mathrm{E}-23$ & \\
\hline Aalb-17,244 & 385 & AalbOR22/N1 ${ }^{\mathrm{c}}$ & AAEL002479 & OR22 & $7 \mathrm{tm} \_6$ & $1.57 \mathrm{E}-31$ & N \\
\hline Aalb-5704 & 402 & AalbOR23 & AAEL001510 & OR23 & $7 \mathrm{tm} \_6$ & $3.88 \mathrm{E}-16$ & \\
\hline Aalb-16,397 & 395 & AalbOR24 & AAEL017557 & OR24 & $7 \mathrm{tm} \_6$ & $3.29 \mathrm{E}-17$ & \\
\hline Aalb-19,286 & 180 & AalbOR24-N1 & AAEL017557 & OR24 & - & - & C \\
\hline Aalb-84,055 & 381 & AalbOR25 & AAEL003629 & OR25 & $7 \mathrm{tm} \_6$ & $2.53 \mathrm{E}-18$ & \\
\hline Aalb-85,326 & 401 & AalbOR26 & AAEL010428 & OR26 & $7 \mathrm{tm} \_6$ & 4.03E-20 & \\
\hline Aalb-88,595 & 384 & AalbOR29 & AAEL001310 & OR29 & $7 \mathrm{tm} \_6$ & $1.08 \mathrm{E}-23$ & N \\
\hline Aalb-17,821 & 384 & AalbOR30 & AAEL010409 & OR30 & $7 \mathrm{tm} \_6$ & $1.95 \mathrm{E}-17$ & \\
\hline Aalb-84,635 & 403 & AalbOR31 & AAEL013217 & OR31 & $7 \mathrm{tm} \_6$ & $9.78 \mathrm{E}-44$ & \\
\hline Aalb-81,229 & 396 & AalbOR33 & AAEL017362 & OR33 & $7 \mathrm{tm} \_6$ & $2.66 \mathrm{E}-36$ & \\
\hline Aalb-4286 & 406 & AalbOR39 & AAEL005489 & OR39 & $7 \mathrm{tm} \_6$ & 4.01E-42 & \\
\hline Aalb-10,769 & 391 & AalbOR42 & AAEL000616 & OR41 & $7 \mathrm{tm} \_6$ & 3.03E-20 & \\
\hline Aalb-80,037 & 386 & AalbOR42-N1 & AAEL003045 & OR42 & $7 \mathrm{tm} \_6$ & $6.57 \mathrm{E}-22$ & \\
\hline Aalb-15,665 & 385 & AalbOR44 & AAEL006465 & OR44 & $7 \mathrm{tm} \_6$ & 6.17E-23 & \\
\hline Aalb-5267 & 381 & AalbOR45 & AAEL000613 & OR45 & $7 \mathrm{tm} \_6$ & $9.44 \mathrm{E}-22$ & N \\
\hline Aalb-18,518 & 207 & AalbOR45-N1 & AAEL000613 & OR45 & $7 \mathrm{tm} \_6$ & $2.04 \mathrm{E}-12$ & $\mathrm{~N}, \mathrm{C}$ \\
\hline Aalb-97,870 & 384 & AalbOR47-N2 & AAEL017079 & OR47 & $7 \mathrm{tm} \_6$ & 1.40E-27 & \\
\hline Aalb-56,511 & 170 & AalbOR47-N3 & AAEL017079 & OR47 & - & - & $\mathrm{N}, \mathrm{C}$ \\
\hline Aalb-7041 & 257 & AalbOR50-N1 & AAEL010426 & OR50 & $7 \mathrm{tm} \_6$ & 4.01E-03 & C \\
\hline Aalb-5118 & 421 & AalbOR52 & AAEL013507 & OR52 & $7 \mathrm{tm} \_6$ & 5.40E-11 & \\
\hline Aalb-70,366 & 395 & AalbOR55 & AAEL010415 & OR55 & $7 \mathrm{tm} \_6$ & $3.88 \mathrm{E}-17$ & \\
\hline Aalb-1114 & 250 & AalbOR59 & AAEL001342 & OR59 & - & - & C \\
\hline Aalb-69,237 & 375 & AalbOR62 & AAEL011796 & OR62 & $7 \mathrm{tm} \_6$ & $4.34 \mathrm{E}-11$ & N \\
\hline Aalb-4058 & 286 & AalbOR63 & AAEL000628 & OR63 & $7 \mathrm{tm} \_6$ & $3.38 \mathrm{E}-24$ & N \\
\hline Aalb-89,176 & 404 & AalbOR63-N2 & AAEL000628 & OR63 & $7 \mathrm{tm} \_6$ & $1.01 \mathrm{E}-23$ & \\
\hline Aalb-696 & 389 & AalbOR66 & AAEL017227 & OR66 & $7 \mathrm{tm} \_6$ & 4.75E-18 & \\
\hline Aalb-47,052 & 386 & AalbOR69 & AAEL001221 & OR69 & $7 \mathrm{tm} \_6$ & $2.53 \mathrm{E}-12$ & \\
\hline Aalb-73,112 & 199 & AalbOR70 & AAEL001224 & OR70 & $7 \mathrm{tm} \_6$ & 2.92E-04 & $\mathrm{N}, \mathrm{C}$ \\
\hline Aalb-10,463 & 385 & AalbOR70-N1 & AAEL001224 & OR70 & $7 \mathrm{tm} \_6$ & 7.75E-19 & \\
\hline Aalb-81,174 & 385 & AalbOR70-N2 & AAEL001224 & OR70 & $7 \mathrm{tm} \_6$ & 2.71E-17 & \\
\hline Aalb-13,202 & 422 & AalbOR71 $1^{c}$ & AAEL017564 & OR18 & $7 \mathrm{tm} \_6$ & $3.75 \mathrm{E}-23$ & \\
\hline Aalb-6552 & 412 & AalbOR72 & AAEL017129 & OR72 & $7 \mathrm{tm} \_6$ & 5.63E-09 & \\
\hline
\end{tabular}


Table 3 Aedes albopictus putative odorant receptor (OR) transcripts (Continued)

\begin{tabular}{|c|c|c|c|c|c|c|c|}
\hline & & & Ae. aegypti or & & Conserv & & \\
\hline Aalb-5361 & 412 & AalbOR72-N1 & AAEL017129 & OR72 & $7 \mathrm{tm} \_6$ & $4.82 \mathrm{E}-06$ & $S$ \\
\hline Aalb-6097 & 225 & AalbOR76 & AAEL013418 & OR76 & $7 \mathrm{tm} \_6$ & $6.51 \mathrm{E}-04$ & $\mathrm{~N}, \mathrm{C}$ \\
\hline Aalb-9669 & 395 & AalbOR79 & AAEL013420 & OR79 & $7 \mathrm{tm} \_6$ & $7.00 \mathrm{E}-07$ & \\
\hline Aalb-3076 & 422 & AalbOR80 & AAEL017221 & OR80 & $7 \mathrm{tm} \_6$ & 2.39E-05 & \\
\hline Aalb-97,462 & 423 & AalbOR81 & AAEL017305 & OR81 & $7 \mathrm{tm} \_6$ & $1.43 \mathrm{E}-08$ & \\
\hline Aalb-18,076 & 353 & AalbOR83c & AAEL000628 & OR63 & $7 \mathrm{tm} \_6$ & $3.74 \mathrm{E}-20$ & N \\
\hline Aalb-83,353 & 410 & AalbOR84 & AAEL004218 & OR85 & 7tm_6 & $1.81 \mathrm{E}-3$ & $\mathrm{~N}$ \\
\hline Aalb-89,654 & 416 & AalbOR85 ${ }^{c}$ & AAEL017043 & OR84 & $7 \mathrm{tm} \_6$ & 5.89E-07 & \\
\hline Aalb-89,563 & 424 & AalbOR87 & AAEL017347 & OR87 & - & - & \\
\hline Aalb-445 & 353 & AalbOR87-N1 & AAEL017347 & OR87 & $7 \mathrm{tm} \_6$ & 8.59E-05 & $\mathrm{N}$ \\
\hline Aalb-89,562 & 288 & AalbOR87-N2 & AAEL017347 & OR87 & - & - & $\mathrm{N}$ \\
\hline Aalb-92,688 & 408 & AalbOR88 & AAEL014197 & OR88 & - & - & \\
\hline Aalb-84,524 & 123 & AalbOR94 & AAEL017201 & OR94 & $7 \mathrm{tm} \_6$ & 2.01E-03 & $\mathrm{N}$ \\
\hline Aalb-53,071 & 172 & AalbOR94-N1 & AAEL017201 & OR94 & - & - & C \\
\hline Aalb-50,344 & 283 & AalbOR99 & AAEL017236 & OR99 & - & - & $\mathrm{N}$ \\
\hline Aalb-96,847 & 415 & AalbOR100 & AAEL011409 & OR100 & - & - & \\
\hline Aalb-26,945 & 210 & AalbOR101 & AAEL017050 & OR101 & - & - & $\mathrm{N}$ \\
\hline Aalb-9171 & 363 & AalbOR102 & AAEL017143 & OR102 & $7 \mathrm{tm} \_6$ & 9.37E-03 & $\mathrm{N}$ \\
\hline Aalb-96,630 & 405 & AalbOR104-N1 ${ }^{c}$ & AAEL016966 & OR104 & $7 \mathrm{tm} \_6$ & $2.24 \mathrm{E}-05$ & \\
\hline Aalb-17,596 & 150 & AalbOR109 & AAEL017028 & OR109 & - & - & $\mathrm{N}$ \\
\hline Aalb-47,815 & 244 & AalbOR110 & AAEL016983 & OR110 & - & - & $\mathrm{N}, \mathrm{C}$ \\
\hline Aalb-97,176 & 421 & AalbOR $111^{c}$ & AAEL017178 & OR105 & $7 \mathrm{tm} \_6$ & 2.97E-03 & \\
\hline Aalb-97,175 & 420 & AalbOR111-N1 ${ }^{c}$ & AAEL017178 & OR105 & - & - & \\
\hline Aalb-82,645 & 418 & AalbOR113 & AAEL017123 & OR113 & - & - & \\
\hline Aalb-84,373 & 157 & AalbOR113-N2 & AAEL017123 & OR113 & - & - & $\mathrm{N}, \mathrm{C}$ \\
\hline Aalb-5235 & 230 & AalbOR115 & AAEL017361 & OR115 & - & - & $\mathrm{N}$ \\
\hline Aalb-45,911 & 169 & AalbOR117-N1 & AAEL017377 & OR117 & - & - & $\mathrm{N}$ \\
\hline Aalb-15,754 & 415 & AalbOR117-N2 & AAEL017377 & OR117 & $7 \mathrm{tm} \_6$ & $6.14 \mathrm{E}-03$ & $\mathrm{~N}$ \\
\hline Aalb-7651 & 160 & AalbOR117-N3 & AAEL017377 & OR117 & - & - & $\mathrm{N}, \mathrm{C}$ \\
\hline Aalb-56,571 & 417 & AalbOR117-N4 & AAEL017377 & OR117 & $7 \mathrm{tm} \_6$ & 4.45E-04 & \\
\hline Aalb-53,133 & 421 & AalbOR121 & AAEL017104 & OR121 & $7 \mathrm{tm} \_6$ & 4.93E-04 & \\
\hline Aalb-16,771 & 406 & AalbOR122 & AAEL013563 & OR122 & - & - & \\
\hline Aalb-71,045 & 376 & AalbOR123 & AAEL017537 & OR123 & $7 \mathrm{tm} \_6$ & $2.41 \mathrm{E}-08$ & \\
\hline Aalb-84,576 & 311 & AalbOR125 & AAEL013893 & OR125 & $7 \mathrm{tm} \_6$ & $1.92 \mathrm{E}-05$ & $\mathrm{~N}$ \\
\hline Aalb-86,662 & 416 & AalbOR-N5 & AAEL017014 & - & $7 \mathrm{tm} \_6$ & 9.49E-05 & \\
\hline Aalb-401 & 413 & AalbOR-N6 & AAEL017014 & - & - & - & $\mathrm{N}$ \\
\hline Aalb-3853 & 414 & AalbOR-N7 & AAEL010669 & - & $7 \mathrm{tm} \_6$ & $1.89 \mathrm{E}-39$ & \\
\hline
\end{tabular}

according to Bohbot and colleagues [64]

${ }^{b} \mathrm{~N}, \mathrm{C}$ : incomplete at $\mathrm{N}$ - and/or C-terminus; S: contains internal stop

cname modified to comply with Lombardo and colleagues [65]

separating them from the two Italian populations. The second component (accounting for $28.4 \%$ of the variation) separates the Athens population from the native Ban Rai population.
SNP variation within the olfactory protein transcripts

The 44 considered OBP transcripts contain 463 SNP loci (including clustered loci). Most were bi-allelic, with only six tri-allelic loci (1.3\%) in six transcripts: AalbOBP22, 


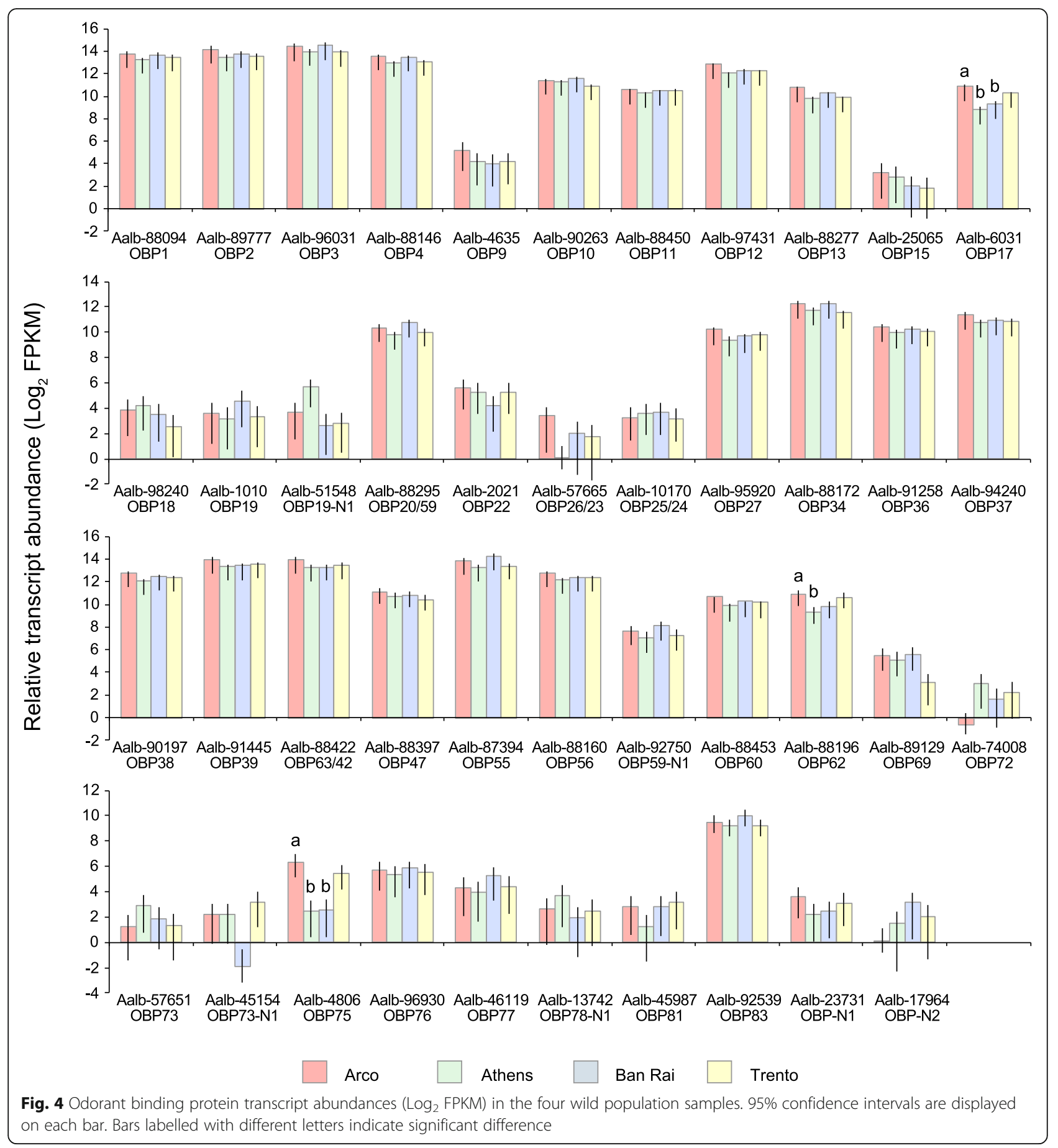

AalbOBP34, AalbOBP69, AalbOBP59-N1, AalbOBP60 and AalbOBP76. Over 18\% (85) of the SNP variants result in non-synonymous substitutions, whereas the remaining variants result in synonymous substitutions. However, as 25 of the non-synonymous substitutions are in the signal-peptide encoding sequences, just under $13 \%$ (60) of the SNPs would result in amino acid substitutions in the mature OBPs (Additional file 12: Table S13).
A total of 1900 SNP loci (including clustered loci) are present in the 78 OR transcripts, 16 of which are triallelic. About 25\% (484) of the SNP variants result in nonsynonymous substitutions (Additional file 13: Table S14). The differentially transcribed AalbOR100 contains 31 SNP loci, of which 13 result in non-synonymous substitutions. Four of these SNP variants are private, three in Ban Rai (two of which are non-synonymous) and one in Athens. The other differentially expressed odorant 


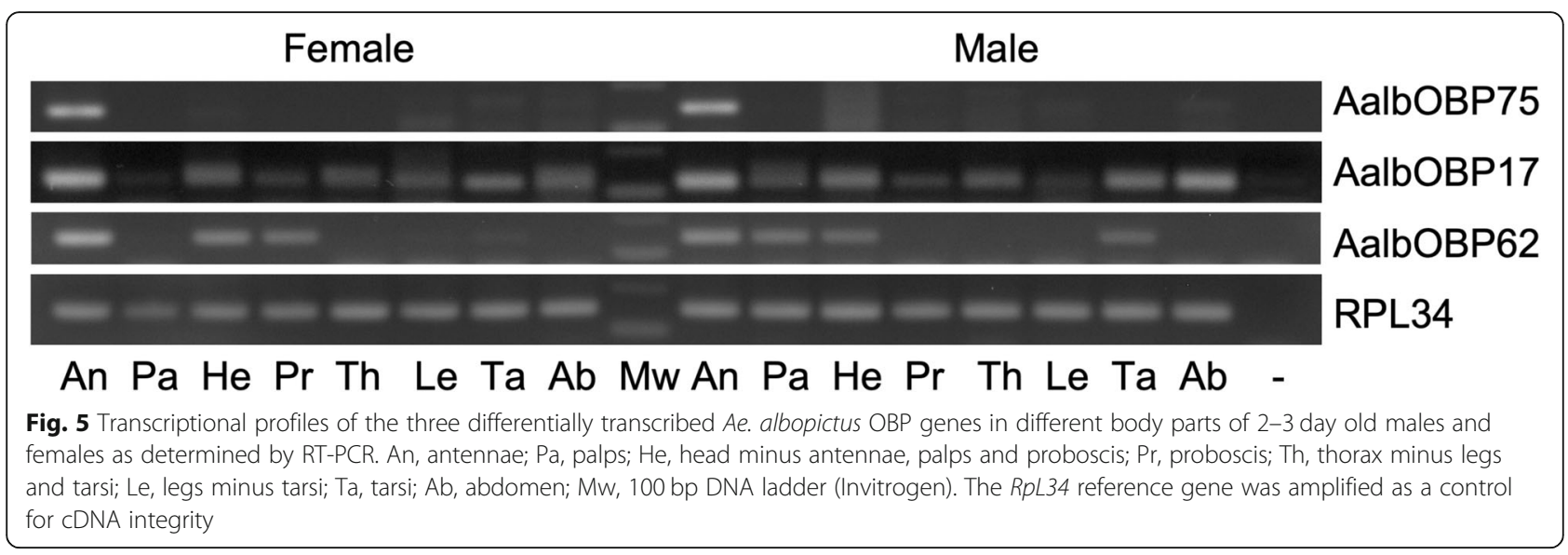

receptor, AalbOR47-N2 contains 19 SNP loci, seven of which are non-synonymous. Three of these SNP variants, including a non-synonymous substitution, were private, all in Ban Rai. In both these ORs, a nonsynonymous substitution was present in the extracellular loop 2 (ECL2) region that has been shown to determine odorant specificity $[69,70]$ (Fig. 8). Of the other 32 OR proteins, for which it was possible to derive the seven transmembrane domain structure, ten contained from one to two non-synonymous substitutions in the ECL2 region. The AalbOR7/ORCO transcript has 23 SNP loci of which only one variant is non-synonymous resulting in the substitution of a Serine with another polar residue, Threonine, in the ORCO-specific insertion on the second intracellular loop (ICL2) of the protein complex $[71,72]$. This substitution is private to the Ban Rai population with a frequency of $16 \%$. The affected amino acid is 51 residues downstream from a calmodulin binding site $(\mathrm{CaM})$ also in ICL2. The ORCO CaM binding site is involved in the phenomenon of sensitization, whereby repetitive subthreshold odour stimulation of olfactory sensory neurons sensitizes the OR/ORCO complex to give a super threshold response, enabling the insect to detect very faint odour traces [73, 74].

In contrast to these highly variable genes, the two arrestin genes (arrestin-1 and arrestin-2) contained respectively 33 and 22 SNP loci. Only one of the SNP loci, in arrestin-1, was non-synonymous resulting in the substitution of a non-polar Phenylalanine with a polar Tyrosine. This polymorphism is within the arrestin $\mathrm{N}$ terminal domain.

Tests of selection for the differentially transcribed $O B P$ and OR transcripts

Evaluation of the ratios of fixed or polymorphic, nonsynonymous/synonymous sites in the differentially transcribed OBP and OR genes, AalbOBP17, AalbOBP75, AalbOBP62, AalbOR47-N2 and AalbOR100, compared with their Ae. aegypti orthologues did not reveal any significant indication of departure from neutral molecular evolution (Fisher's Exact test $P>0.05$, McDonaldKreitman test [53]).

\section{Discussion}

Here we provide an overview of the vast reservoir of transcribed genes coding for sensory related functions in the antennae of wild mature adult mosquitoes collected in different ecogeographic areas. The information present in antennal transcriptomes is important both from an evolutive and an applicative point of view. Indeed, it is the basis to analyse and interpret different biological traits related to the behaviour and reproduction of this highly invasive mosquito. Another biologically important result is the high transcriptional variability of the sensory related genes among the wild populations of this mosquito. This variability may reflect the genetic plasticity that characterizes this mosquito and that has supported its high invasive potential. We have identified functional gene categories that display population-specific differential transcription levels. Given the public health impact of this mosquito, the information that we provide may prove important in the implementation of control methods such as the Sterile Insect Technique (SIT).

\section{Functional gene categories displaying population-specific} differential transcription levels

\section{Sound, vision and odorant perception terms}

Enrichment of transcripts of genes associated with the perception of sound was evident in the Greek Athens and Thai Ban Rai samples in the comparisons with the Italian Arco sample. These transcripts included several dynein intermediate and heavy chain homologues and a nompC (no mechanoreceptor potential C) homologue which in Drosophila are expressed in chordotonal neurons. These are required for mechanical amplification of vibrations and mechanosensory function within the Johnston's organ, a chordotonal organ near the base of 


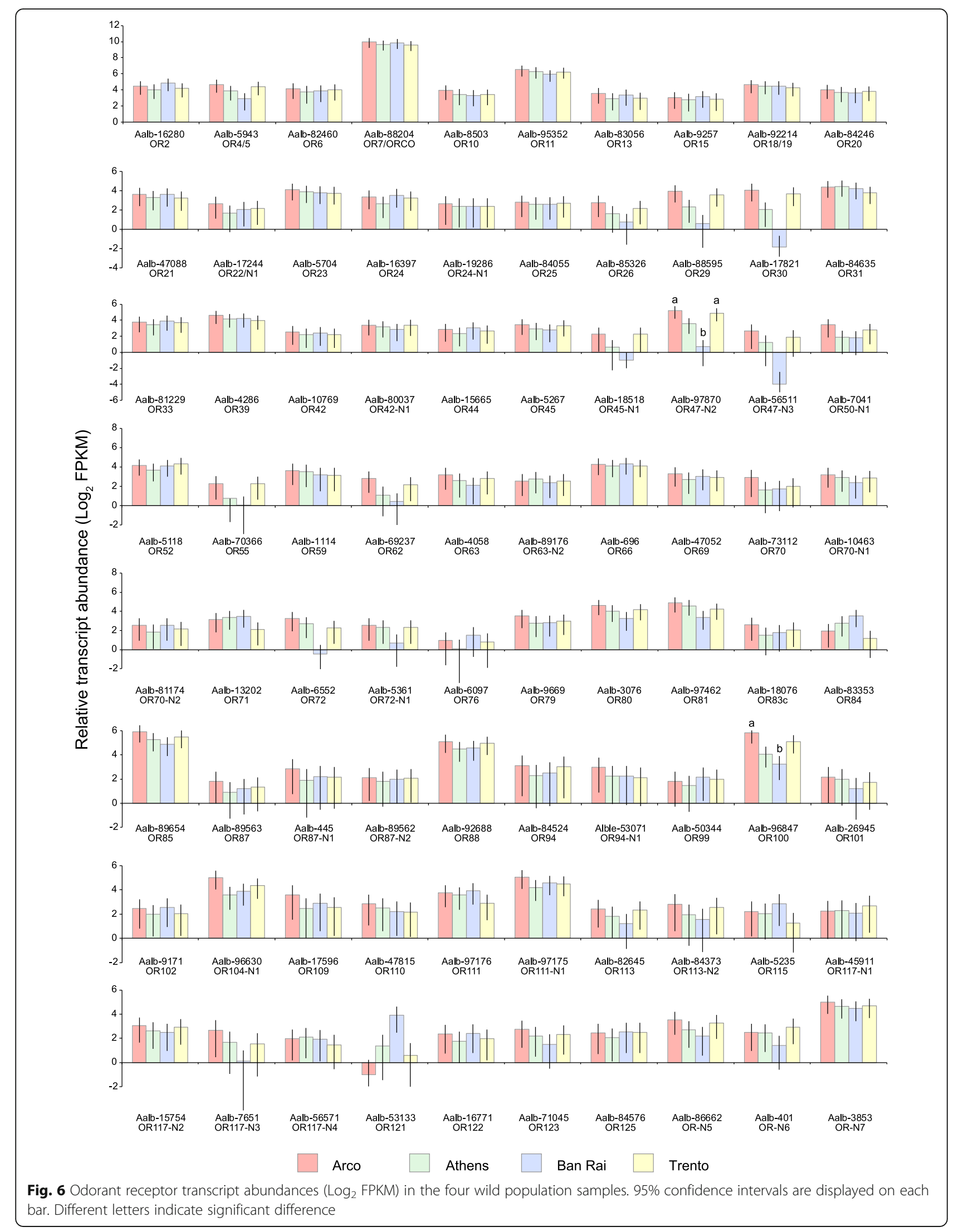




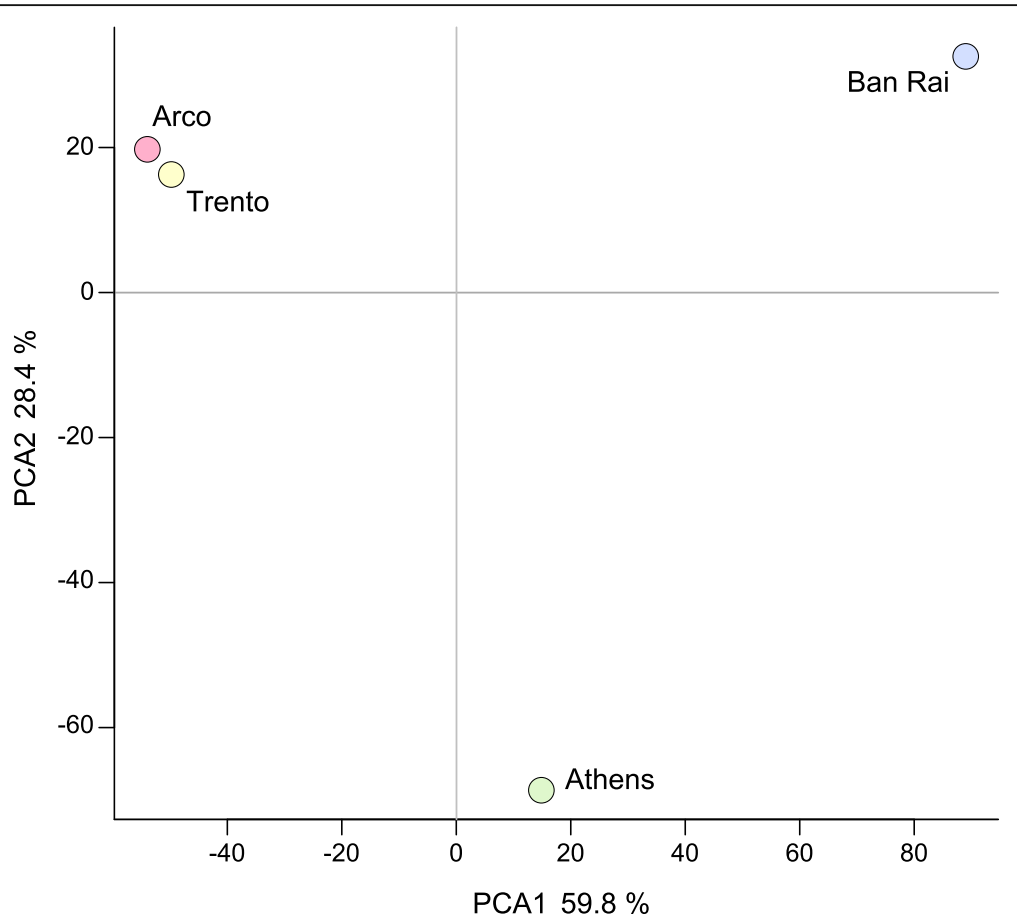

Fig. 7 Principal Component Analysis based on biallelic SNP frequencies illustrating the relationships between the four population samples

the antenna [75]. In the mosquito, the Johnston's organs are especially well developed in the male [76], where they are acutely sensitive to the wing-beat frequency of the female mosquito - a cue that is essential for mating success [77]. These organs may have additional functions such as gravitactic behaviour and the detection of air currents during flight [78].

The enriched regulation and maintenance of photoreception GO category in Arco compared to Trento and Athens involved homologues of arrestin-1 and arrestin-2 of An. gambiae. Arrestins are involved in the desensitization of both visual and olfactory transduction pathways where they regulate $\mathrm{G}$ protein-coupled receptors $[79,80]$. Given the antennal origin of the transcriptome, these arrestins may be regulating olfactory signalling by coupling to ORs [64]. Thus, this GO-term may relate to olfaction rather than to vision.

Transcripts related to the perception of smell were enriched in Ban Rai compared to Arco. These included homologues of the sensory neuron membrane protein 1 (SNMP1), a member of a family of SNMPs originally identified in Lepidoptera where they are antennalspecific and are associated with pheromone specific sensilla [81]. In D. melanogaster SNMP1 is essential for the detection of the male-specific pheromone cis-vaccenyl acetate [82].

The apparent absence of many Ae. aegypti OBP, OR and GR homologues is not unexpected, as many will be associated with chemosensory tissues other than the antennae, such as the maxillary palps, proboscis and other parts of the body [83]. Indeed, the OBP and OR repertoires here identified differ slightly compared to those recently described in Ae. albopictus by Lombardo and colleagues [65], that analysed both antennae and maxillary palps (Additional file 8: Table S10). Other genes may be limited to larval stages [63], or different physiological states (e.g. after blood-feeding).

Among the identified OBP transcripts, three were significantly up-regulated in the Arco population sample in the comparisons with both the Athens and Ban Rai samples (AalbOBP17, AalbOBP75) or with Athens (AalbOBP62). The Classic OBP AalbOBP17 appears to be transcribed in numerous body compartments in Ae. albopictus whereas its orthologue AaegOBP17/ AAEL004339 is mainly transcribed in the antennae of females and, to a lesser extent, in males [84]. The Plus-C OBP, AalbOBP75, appears to be transcribed only in the antennae of both sexes, whereas its Ae. aegypti orthologue (AaegOBP75/AAEL011483) is mainly transcribed in the female antennae (where blood-feeding appears to up-regulate its transcription), in maxillary palps and to a lesser extent in the female rostrum and male antennae [84]. Furthermore, AaegOBP75 is enriched in the salivary glands of virally naïve females [85]. The enriched AalbOBP62, another Plus-C OBP, has a transcriptional profile that is congruent with that shown by Deng and colleagues [86] and with that of its Ae. aegypti orthologue (AaegOBP62/AAEL015566) [84]. Interestingly, 


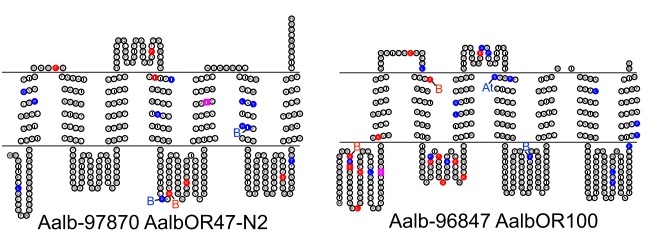

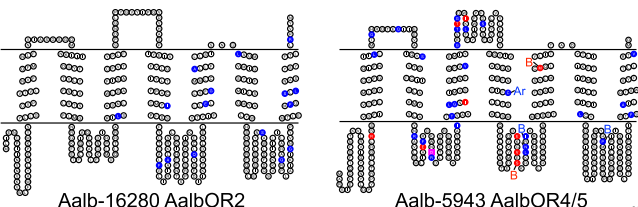

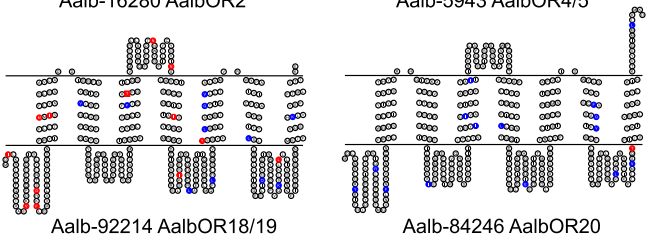
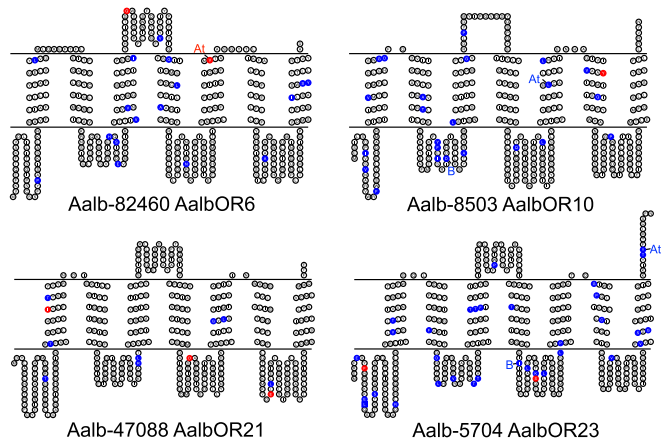

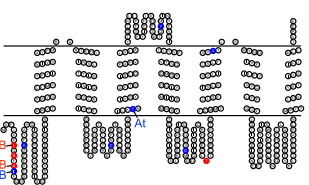

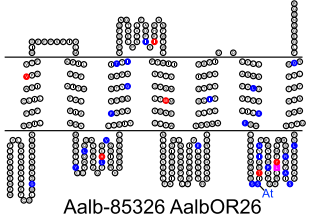

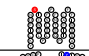
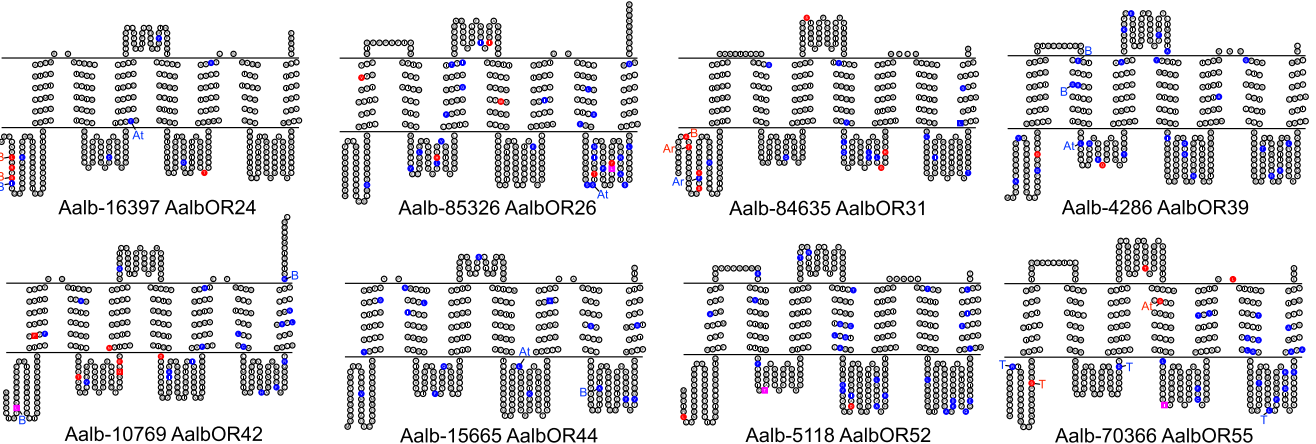

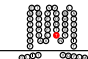

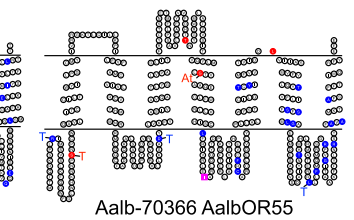

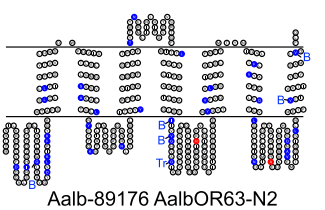
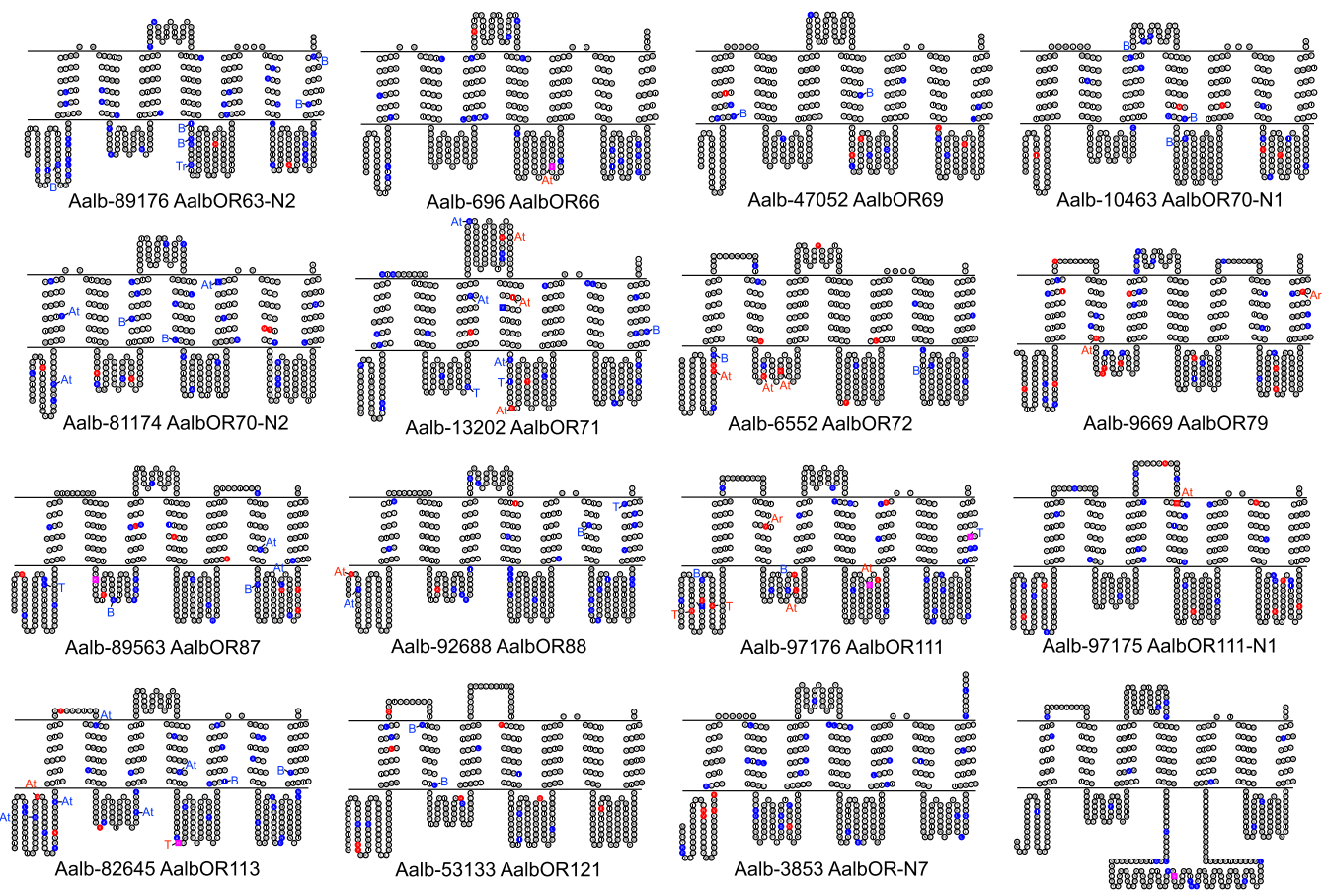

Fig. 8 (See legend on next page.) 
(See figure on previous page.)

Fig. 8 Snakeplots of Ae. albopictus ORs. Amino acids are coloured to indicate synonymous (blue), non-synonymous (red) and the presence of both non-synonymous and synonymous substitutions within the codon (magenta). Coloured circles indicate codons with one SNP locus, squares indicate codons with two or more SNP loci. The position of SNP variants that are private to one population sample $(B=B a n$ Rai, $A t=A t h e n s, A r=$ Arco, $T=$ Trento) are indicated

AaegOBP62 transcription increased in Ae. aegypti females $24 \mathrm{~h}$ after infection with yellow fever virus [87].

At least 83 ORs are transcribed in the antennae of Ae. aegypti [63], a number similar to that identified in the current study (at least 78). Due to its highly-fragmented state, the published draft Fellini genome sequence [60] was only marginally useful for assembly of the many OBP- and OR-like fragments. In contrast, the Chinese strain genomic sequence [61], though less fragmentary, appears to be rather redundant. Transcripts of two ORs, AalbOR100 and AalbOR47-N2, were found to be upregulated in one or both Italian populations compared to the Ban Rai population. Both Ae. aegypti orthologues of these ORs are enriched in female compared to male antennae [84] and are upregulated in domestic forms that prefer to bite humans compared to forest forms that prefer guinea pigs [88], suggesting that they may be involved in the perception of odours related to host detection.

The identification of the highly conserved gustatory receptor GR1 orthologue (98\% identity with AaegGR1) in the antennae is noteworthy, as in Ae. aegypti, this gene is expressed exclusively in the maxillary palps [89], where it acts as a $\mathrm{CO}_{2}$ receptor. Carbon dioxide is an important cue that emanates from potential blood-meal hosts and guides females over relatively long distances and, together with heat and other olfactory cues, stimulates blood feeding.

\section{Iron-ion binding terms}

The iron-ion-binding GO category was enriched in the Athens sample compared to Trento and Ban Rai, with 14 cytochrome P450 transcripts up-regulated, together with glutamate synthase, a carboxy/choline esterase alpha esterase, $\mathrm{ABC}$ transporter, aconitase and transferrin. Aconitase or iron regulatory protein 1 (IRP1) controls iron uptake by cells via the transferrin receptor and iron storage within cells as ferritin [90]. Transferrins have high affinities for iron and are involved in metabolism, immunity and development. Transferrins are upregulated following bacterial or parasite infection and sequester iron from pathogens $[85,91]$. Several studies have shown that transferrins are down-regulated in individuals infected with CHIKV and DENV-2 infections $[87,92]$, and it has been suggested that this may favour viral replication and survival, perhaps due to subversion of the insect's pathways by the arboviruses [93].
Numerous cytochrome P450 transcripts were more abundant in the Athens sample. Cytochrome P450s are one of the largest and oldest gene superfamilies in insects and are involved in the metabolism of many endogenous and xenobiotic compounds including the detoxification of insecticides [94]. Indeed, at least four of the more abundant cytochrome P450s (CYP6Z8, CYP6Z9, CYP9J26 \& CYP9J9) have been implicated in Deltamethrin and or Permethrin resistance in Ae. aegypti [95]. The extremely high abundance of Carboxy/ cholinesterase alpha esterase, (AealbCCEae3a) in the Athens population (59-fold) compared to the other population samples is surprising. But, given that the Ae. albopictus CCEae3a gene has been implicated in temephos resistance, an organophosphate (OP) insecticide that has been used extensively as a larvicide in the Athens area for many years $[95,96]$, it is probable that this upregulation, due to gene copy number amplification, is more likely related to insecticide resistance than to iron-ion-binding. Recently a study of population samples from 16 countries found evidence of amplification only in Athens and Florida [58]. Two independent amplification types were identified, one involving coamplification of the linked CCEae3a and CCEae6a genes, and individuals with this type in Athens and Florida shared the same haplotype. The second amplification type involves only the CCEae3a gene and was found only in Florida.

\section{Nitrogen assimilation terms}

Another up-regulated category related to nitrogen assimilation included glutamate synthase activity. Ammonia is metabolised, primarily in the fat body, by glutamate synthase with the production of glutamine and of proline that can be used as an energy source for flight $[97,98]$. Why this term should be enriched in the Athens and Ban Rai samples compared to the two Italian samples is not clear. It should, however be mentioned that the adventive Athens mosquitoes share a common demographic history with those from Thailand [18].

\section{Viral infection terms}

The enrichment of viral infection terms in the two geographically close northern Italian populations related to the polyprotein gene of an Aedes flavivirus (AeFV). This virus was initially identified in Japanese populations of Ae. albopictus and Ae. flavopictus collected in 20032004 [66] and subsequently in Ae. albopictus from 
Missouri, USA in 2011 [67] and Ae. albopictus from Curitiba, Brazil in 2016 [99]. AeFV appears to be an insect-specific flavivirus (ISF) with no known vertebrate host, and it has been shown to be transmitted vertically to progeny [67]. AeFV has been shown to be widely distributed in Ae. albopictus in northern Italy including the Arco and Trento populations with an estimated viral infection prevalence of $16.84 \%$ in the Trentino province [100-102] and 3.12\% in Veneto [102]. The northern Italian Ae. albopictus populations were established through a mixture of genomes originating from introductions from North America, the Pacific area and Japan/Southeast Asia [18, 19, 103, 104]. The Athens population, however, appears to be the result of a recent introduction directly from Thailand $[18,19]$. The differences in the AeFV transcript abundances, high in the two Italian populations and practically absent in the Thai and Athens samples, may thus reflect the historical demographic relationships between the population samples. However, the possibility of de novo flavivirus infection of the Italian populations cannot be excluded. Whether these transcripts represent viral integrations in the mosquito genome or infections needs additional study. Should they derive from infections, it would suggest that the viral infection is maintained in the derived populations despite the bottleneck events that accompany new colonization events and the viral prevalence persists over numerous generations.

The presence of these non-pathogenic ISFs within a mosquito host may have important implications for disease dynamics [102]. It has been postulated that ISF infections may reduce the ability of the mosquito to harbour and transmit other related disease-causing viruses as a result of competition between the viruses for the host, due to 'super-infection exclusion' $[105,106]$. For instance, the higher prevalence of AeFV in Trentino compared to populations from Veneto may be correlated with the apparent lower incidence of West Nile virus and Usutu virus in the Trentino with respect to Veneto [102].

\section{A vast repertoire of SNPs is in present in the antennal transcriptomes of wild populations}

The identification of nearly one million SNP loci represents a useful addition to the arsenal of high-resolution molecular markers available in this species (reviewed in [16, 18]). Indeed, unlike Anopheles gambiae [107] and Aedes aegypti [108], few high-resolution molecular markers such as single-nucleotide polymorphisms (SNPs) were previously available for Ae. albopictus [16, 21-23]. The availability of a large number of SNP markers distributed throughout the genome is advantageous as it permits the identification of fine-scale differentiation between populations. Furthermore, given their transcriptomic origin, these SNPs may represent markers of selective pressure and adaptations that have occurred during the colonization of new habitats. The SNP densities in the four population samples ranged from 7.30 SNPs per kilobase in Arco to 10.35 in Ban Rai. The significantly higher SNP density and frequency of private SNP variants in Ban Rai (Additional file 13: Table S14) is a further indication of the native status of this population. SNP loci were abundant in the OBP and OR transcripts with numerous non-synonymous variants that may, depending on their position and the properties of the amino acids involved, change the affinity of the protein for its ligand.

\section{Conclusions}

The high degree of inter-population transcriptional diversity highlights, at the functional level, the remarkable genetic flexibility of this mosquito species. We can hypothesize that the differential expression of genes, including those involved in sensory perception, in different populations may enable Ae. albopictus to exploit different environments and hosts. Furthermore, the discovery of this vast reservoir of transcriptional variability can help us interpret, at the molecular/functional levels, some of the biological traits that support its invasive potential.

From an evolutive point of view, in relation to the invasion process, the native Thai population appears to be the most differentiated with respect to the adventive Mediterranean populations, which are representative of some of the oldest invasion events outside Asia [18]. Among these Mediterranean populations, Athens is quite apart and is unique in sharing with Thailand a high number of enriched transcripts. This may be expected considering its direct demographic origin from Thailand [18, 19], while the origins of the northern Italian Arco and Trento populations is more complex $[10,18,21]$. Thus, we can speculate that ancestry, consequent to the demographic histories of populations, may affect the transcriptional profiles of genes related to sensory perception, which in turn may influence their behaviour.

A large number of SNP loci are also present in these transcripts across the considered populations. SNP variation within these genes may highlight the effect of the colonization processes and may reveal details of Ae. albopictus movement and gene flow patterns. This is evident in the analysis of SNP frequencies in the four considered populations where the relationships between the native Thai population, the adventive Athens and Italian populations was supported. Here, given the antennal origin of the libraries, we have highlighted SNP variation in a subset of genes involved in chemoreception, but clearly the resource we have developed can be applied to the study of a vast array of functional categories. These transcriptome-derived SNPs represent an important addition to the largely non-coding regionderived SNPs previously identified [10, 21-23]. 


\section{Supplementary information}

Supplementary information accompanies this paper at https://doi.org/10. 1186/s12864-020-06956-6.

Additional file 1: Table S1. Primers used for RT-PCR analyses.

Additional file 2: Table S2. Primers used for qRT-PCR analyses.

Additional file 3: Figure S1. Molecular function (level 3) Gene Ontology classification of the transcripts that were differentially abundant in the different population libraries.

Additional file 4: Table S3. Significantly over- and under-represented gene ontology categories among transcripts that showed changes in abundance in Ban Rai compared to Athens. Table S4. Significantly overand under-represented gene ontology categories among transcripts that showed changes in abundance in Ban Rai compared to Trento. Table S5. Significantly over- and under-represented gene ontology categories among transcripts that showed changes in abundance in Ban Rai compared to Arco. Table S6. Significantly over- and under-represented gene ontology categories among transcripts that showed changes in abundance in Athens compared to Trento. Table S7. Significantly over- and under-represented gene ontology categories among transcripts that showed changes in abundance in Athens compared to Arco. Table S8. Significantly over- and under-represented gene ontology categories among transcripts that showed changes in abundance in Arco compared to Trento.

Additional file 5: Figure S2. Validation of RNA-seq derived changes in transcript abundance with quantitative real-time RT-PCR for six genes. The $\log _{2}$ transformed ratios of FPKM in comparisons between population samples are plotted against the corresponding $\log _{2}$ transformed relative transcript abundance values obtained with real-time $q R T-P C R$ ratios for six genes (Additional file 6: Table S9). The Pearson correlation coefficient, $r=0.912$, is highly significant $(P=3.6 \mathrm{E}-05)$.

Additional file 6: Table S9. Correlation of RNAseq transcript abundance ratios with real-time qRT-PCR ratios for six genes $\left(\log _{2}\right.$ transformed).

Additional file 7: Figure S3. Phylogenetic relationships of OBP proteins from Ae. albopictus and Ae. aegypti. The unrooted maximum likelihood (log likelihood $=-27,485)$ tree was inferred using the W\&G model (Whelan and Goldman 2001) with a discrete Gamma distribution. Bootstrap values greater than 50\% (1000 replications) are shown. OBPs belonging to the Classic, Plus- $\mathrm{C}$ and Two domains subfamilies are highlighted.

Additional file 8: Table S10. Comparison of the odorant binding protein transcripts identified against the Lombardo (2017) transcripts. Table S11. Comparison of the odorant receptor transcripts identified against the Lombardo (2017) transcripts.

Additional file 9: Figure S4. Phylogenetic relationships of OR proteins from Ae. albopictus and Ae. aegypti. The unrooted maximum likelihood (log likelihood $=-84,078$ ) tree was inferred using the JTT model (Jones et al. 1992) with a discrete Gamma distribution and amino acid frequencies (JTT $+G+F)$. Bootstrap values greater than 50\% (1000 replications) are shown.

Additional file 10: Table S12. Aedes albopictus putative odorant receptor (GR) transcripts (BLASTP against NR database).

Additional file 11: Figure S5. Relationship between the number of polymorphic sites per kilobase and read depth (FPKM) for the four population samples. For clarity, the $x$-axes are truncated at FPKM $=2000$, which precludes the vision of less than $0.09 \%$ of the data points. Trendlines are plotted on each of the graphs.

Additional file 12: Table S13. Frequencies of synonymous and nonsynonymous SNP variants in the OBP transcripts.

Additional file 13: Table S14. Frequencies of synonymous and nonsynonymous SNP variants in the OR transcripts.

\section{Abbreviations}

aa: Amino acid; ABC: ATP-binding cassette; ABySS: Assembly by short sequences; AeFV: Aedes flavivirus; ANOVA: Analysis of Variance; BAM: Binary
Alignment Map; BLAST: Basic Local Alignment Search Tool; BLOSUM: BLOcks SUbstitution Matrix; bp: Base pair; CaM: Calmodulin binding site; CAP3: Contig assembly program Version 3; CDD: Conserved Domains Databases; cDNA: complementary DNA; CDS: Coding sequence; CHIKV: Chikungunya virus; $\mathrm{CO}_{2}$ : Carbon dioxide; dCAS: Desktop cDNA Annotation System; DENV-2: Dengue virus serotype 2; DNA: Deoxyribonucleic acid; DNAse: Deoxyribonuclease; DnaSP: DNA sequence polymorphism analysis; FDR: False discovery rate; FPKM: Fragments Per Kilobase of transcript per Million mapped reads; FS: Fisher Strand; G0: Generation zero;

GATK: Genome Analysis ToolKit; GO: Gene ontology; GR: Gustatory receptor; ICL2: Second intracellular loop; IGV: Integrative Genomics Viewer; IRP1: Iron regulatory protein 1; ISF: Insect-specific flavivirus; KOG: EuKaryotic Orthologous Groups; M: Million; MAFFT: Multiple Alignment using Fast Fourier Transform; MEGA: Molecular Evolutionary Genetics Analysis; MEMS AT3: MEMbrane protein Structure And Topology Version 3; mRNA: Messenger ribonucleic acid; NCBI: National Center for Biotechnology Information; OBP: Odorant binding protein; OP: Organophosphate; OR: Odorant receptor; ORCO: Odorant receptor coreceptor; ORF: Open reading frame; PCA: Principal component analysis; PCR: Polymerase chain reaction; Pfam: Protein families database; qRT-PCR: Quantitative reverse transcription polymerase chain reaction; RNA: Ribonucleic acid; SIT: Sterile Insect Technique; SMART: Simple Modular Architecture Research Tool; SNMP: Sensory neuron membrane protein; SNP: Single nucleotide polymorphism; TOPO2: Topoisomerase based cloning vector

\section{Acknowledgements}

We thank Pradya Somboon (Chiang Mai University, Thailand), George Koliopoulos and Dionyssia Maselou (Benaki Phytopathological Institute, Greece) and Gabriella Tait for Aedes albopictus eggs collections. This study was benefitted from discussions at International Atomic Energy Agency funded meetings for the Coordinated Research Projects "Comparing Rearing Efficiency and Competitiveness of Sterile Male Strains Produced by Genetic, Transgenic or Symbiont-based Technologies" (D42016) and "Comparing Rearing Efficiency and Competitiveness of Sterile Male Strains Produced by Genetic, Transgenic or Symbiont-based Technologies" and "Development of attractants for male trapping and the evaluation of male quality and sexual capacity in Aedes albopictus" (D44002). The research was performed within the Dept. of Biology and Biotechnology 'L. Spallanzani' recognized within the "Dipartimenti di Eccellenza Programme (2018-2022)" by the Italian Ministry of Education, University and Research (MIUR). This work utilized the computational resources of the NIH HPC Biowulf cluster (http://hpc.nih.gov).

\section{Authors' contributions}

LMG: conceived, planned experiments, data collection, analysis and manuscript preparation; MM and DC: data collection, analysis and manuscript revision; TN: sequencing and manuscript revision; DL: analysis and manuscript revision; JMR: performed the transcriptome assembly, CDS extraction and part of the annotation; ARM and GG: conceived, planned experiments and manuscript preparation; All authors read and approved the final manuscript.

\section{Funding}

This research is part of the EU-FP7 Research Infrastructures project (INFRAVEC ) Grant N 228421 and was partially supported by the Italian Ministry of Health (RF-2010-2318965) and the Fondazione Banca del Monte di Lombardia (Pavia, Italy). JMCR was supported by the Intramural Research Program of the National Institute of Allergy and Infectious Diseases (Vector-Borne Diseases: Biology of Vector Host Relationship, Z01 AI000810-18). An INFRAVEC Call for access to RNAseq facilities was awarded to LMG. The funding bodies played no role in the design of the study and collection, analysis and interpretation of the data and in writing the manuscript.

\section{Availability of data and materials}

The sequence data generated during this study is deposited on the NCBI Sequence Read Archive, under BioProject ID PRJNA602498 (Accessions SAMN13896421-SAMN13896424). The transcript assembly, abundance and SNP data have been submitted to the Open Science Framework data repository, (https://doi.org/10.17605/OSF.IO/SJBVT). 


\section{Ethics approval and consent to participate}

This study did not include the use of humans or other vertebrate animals. No ethics approval is required for experimentation of the study organism, Aedes albopictus. The insect samples were received and maintained in accordance with European Union rules (Regulation 2017/625/EU and Directive 2010/63/EU).

\section{Consent for publication}

Not Applicable.

\section{Competing interests}

Giuliano Gasperi is a member of the BMC Genomics editorial board. The other authors declare that they have no competing interests.

\section{Author details}

'Department of Biology and Biotechnology, University of Pavia, Pavia, Italy. ${ }^{2}$ Department of Genetic Medicine and Development, University of Geneva Medical School, and Swiss Institute of Bioinformatics, Geneva, Switzerland. ${ }^{3}$ Department of Vector Biology, Liverpool School of Tropical Medicine, Liverpool, UK. ${ }^{4}$ Department of Life Sciences, Imperial College London, London, UK. ${ }^{5}$ NIAID, Laboratory of Malaria and Vector Research, NIH, Rockville, MD 20852, USA.

Received: 8 May 2020 Accepted: 27 July 2020

Published online: 07 August 2020

\section{References}

1. Invasive Species Specialist Group I: Global Invasive Species Database. 2015

2. Mogi M, Armbruster P, Tuno N, Campos R, Eritja R. Simple indices provide insight to climate attributes delineating the geographic range of Aedes albopictus (Diptera: Culicidae) prior to worldwide invasion. J Med Entomol. 2015;52(4):647-57.

3. Delatte H, Gimonneau G, Triboire A, Fontenille D. Influence of temperature on immature development, survival, longevity, fecundity, and gonotrophic cycles of Aedes albopictus, vector of chikungunya and dengue in the Indian Ocean. J Med Entomol. 2009:46(1):33-41.

4. Medlock JM, Hansford KM, Schaffner F, Versteirt V, Hendrickx G, Zeller $H$, Van Bortel W. A review of the invasive mosquitoes in Europe: ecology, public health risks, and control options. Vector Borne Zoonotic Dis. 2012;12(6):435-47.

5. Kraemer MU, Sinka ME, Duda KA, Mylne AQ, Shearer FM, Barker CM, Moore CG, Carvalho RG, Coelho GE, Van Bortel W, et al. The global distribution of the arbovirus vectors Aedes aegypti and Ae. albopictus. Elife. 2015;4:e08347.

6. Carvalho RG, Lourenço-de-Oliveira R, Braga IA. Updating the geographical distribution and frequency of Aedes albopictus in Brazil with remarks regarding its range in the Americas. Mem Inst Oswaldo Cruz. 2014; 109(6):787-96

7. Cornel AJ, Hunt RH. Aedes albopictus in Africa? First records of live specimens in imported tires in Cape Town. J Am Mosq Control Assoc. 1991; 7(1):107-8.

8. Schmidt CA, Comeau G, Monaghan AJ, Williamson DJ, Ernst KC. Effects of desiccation stress on adult female longevity in Aedes aegypti and $\mathrm{Ae}$. albopictus (Diptera: Culicidae): results of a systematic review and pooled survival analysis. Parasit Vectors. 2018;11(1):267.

9. Lounibos LP, Kramer LD. Invasiveness of Aedes aegypti and Aedes albopictus and vectorial capacity for chikungunya virus. J Infect Dis. 2016;214(suppl 5):S453-8.

10. Sherpa S, Guéguen M, Renaud J, Blum MGB, Gaude T, Laporte F, Akiner M, Alten B, Aranda C, Barre-Cardi H, et al. Predicting the success of an invader: niche shift versus niche conservatism. Ecol Evol. 2019;9(22):12658-75.

11. Poelchau MF, Reynolds JA, Elsik CG, Denlinger DL, Armbruster PA. Deep sequencing reveals complex mechanisms of diapause preparation in the invasive mosquito, Aedes albopictus. Proc Biol Sci. 2013:280(1759):1-9.

12. Bonizzoni M, Gasperi $G$, Chen $X$, James AA. The invasive mosquito species Aedes albopictus: current knowledge and future perspectives. Trends Parasitol. 2013;29(9):460-8.

13. Kraemer MUG, Reiner RC, Brady OJ, Messina JP, Gilbert M, Pigott DM, Yi D, Johnson K, Earl L, Marczak LB, et al. Past and future spread of the arbovirus vectors Aedes aegypti and Aedes albopictus. Nat Microbiol. 2019;4(5):854-63.
14. Cunze S, Kochmann J, Koch LK, Klimpel S. Niche conservatism of Aedes albopictus and Aedes aegypti - two mosquito species with different invasion histories. Sci Rep. 2018;8(1):7733.

15. Achee NL, Grieco JP, Vatandoost H, Seixas G, Pinto J, Ching-Ng L, Martins AJ, Juntarajumnong W, Corbel V, Gouagna C, et al. Alternative strategies for mosquito-borne arbovirus control. PLoS Negl Trop Dis. 2019;13(1):e0006822.

16. Goubert C, Minard G, Vieira C, Boulesteix M. Population genetics of the Asian tiger mosquito Aedes albopictus, an invasive vector of human diseases. Heredity (Edinb). 2016;117(3):125-34.

17. Manni M, Gomulski LM, Aketarawong N, Tait G, Scolari F, Somboon P, Guglielmino CR, Malacrida AR, Gasperi G. Molecular markers for analyses of intraspecific genetic diversity in the Asian Tiger mosquito, Aedes albopictus. Parasit Vectors. 2015:8:188

18. Manni M, Guglielmino CR, Scolari F, Vega-Rúa A, Failloux AB, Somboon P, Lisa A, Savini G, Bonizzoni M, Gomulski LM, et al. Genetic evidence for a worldwide chaotic dispersion pattern of the arbovirus vector, Aedes albopictus. PLoS Negl Trop Dis. 2017;11(1):e0005332.

19. Vega-Rúa A, Marconcini M, Madec Y, Manni M, Carraretto D, Gomulski LM, Gasperi G, Failloux AB, Malacrida AR. Vector competence of Aedes albopictus populations for chikungunya virus is shaped by their demographic history. Commun Biol. 2020;3(1):326.

20. Sherpa S, Blum MGB, Després L. Cold adaptation in the Asian tiger mosquito's native range precedes its invasion success in temperate regions. Evolution. 2019;73(9):1793-808.

21. Sherpa S, Blum MGB, Capblancq T, Cumer T, Rioux D, Després L. Unravelling the invasion history of the Asian tiger mosquito in Europe. Mol Ecol. 2019; 28(9):2360-77.

22. Kotsakiozi P, Richardson JB, Pichler V, Favia G, Martins AJ, Urbanelli S, Armbruster PA, Caccone A. Population genomics of the Asian tiger mosquito, Aedes albopictus: insights into the recent worldwide invasion. Ecol Evol. 2017;7(23):10143-57.

23. Pichler V, Kotsakiozi P, Caputo B, Serini P, Caccone A, Della Torre A. Complex interplay of evolutionary forces shaping population genomic structure of invasive Aedes albopictus in southern Europe. PLoS Negl Trop Dis. 2019;13(8):e0007554

24. Clements AN. The biology of mosquitoes, vol. Vol 2, sensory reception and behaviour. Oxon: CABI Pub; 1999.

25. Rinker DC, Pitts RJ, Zhou X, Suh E, Rokas A, Zwiebel LJ. Blood meal-induced changes to antennal transcriptome profiles reveal shifts in odor sensitivities in Anopheles gambiae. Proc Natl Acad Sci U S A. 2013;110(20):8260-5.

26. Franco TA, Oliveira DS, Moreira MF, Leal WS, Melo AC. Silencing the odorant receptor co-receptor RproOrco affects the physiology and behavior of the Chagas disease vector Rhodnius prolixus. Insect Biochem Mol Biol. 2016;69: 82-90.

27. Tallon AK, Hill SR, Ignell R. Sex and age modulate antennal chemosensoryrelated genes linked to the onset of host seeking in the yellow-fever mosquito, Aedes aegypti. Sci Rep. 2019;9(1):43

28. Robinson A, Busula AO, Voets MA, Beshir KB, Caulfield JC, Powers SJ, Verhulst NO, Winskill P, Muwanguzi J, Birkett MA, et al. Plasmodiumassociated changes in human odor attract mosquitoes. Proc Natl Acad Sci U S A. 2018;115(18):E4209-18.

29. Clark JT, Ray A. Olfactory mechanisms for discovery of odorants to reduce insect-host contact. J Chem Ecol. 2016;42(9):919-30.

30. Raji Jl, DeGennaro M. Genetic analysis of mosquito detection of humans. Curr Opin Insect Sci. 2017;20:34-8.

31. Valavanidis A, Vlachogianni T. The most important and urgent environmental problems in Greece in the last decade (2000-2010) [http://1 95.134.76.37/scinews/Reports/Rep_Env_problems2000-10.htm]. Accessed 6 Mar 2019.

32. Schäffer AA, Nawrocki EP, Choi Y, Kitts PA, Karsch-Mizrachi I, McVeigh R. VecScreen_plus_taxonomy: imposing a tax(onomy) increase on vector contamination screening. Bioinformatics. 2018;34(5):755-9.

33. Simpson JT, Wong K, Jackman SD, Schein JE, Jones SJ, Birol I. ABySS: a parallel assembler for short read sequence data. Genome Res. 2009;19(6): 1117-23.

34. Xie Y, Wu G, Tang J, Luo R, Patterson J, Liu S, Huang W, He G, Gu S, Li S, et al. SOAPdenovo-trans: de novo transcriptome assembly with short RNASeq reads. Bioinformatics. 2014;30(12):1660-6.

35. Grabherr MG, Haas BJ, Yassour M, Levin JZ, Thompson DA, Amit I, Adiconis X, Fan L, Raychowdhury R, Zeng Q, et al. Full-length transcriptome assembly from RNA-Seq data without a reference genome. Nat Biotechnol. 2011;29(7):644-52. 
36. Karim S, Singh P, Ribeiro JM. A deep insight into the sialotranscriptome of the gulf coast tick, Amblyomma maculatum. PLoS One. 2011;6(12):e28525.

37. Guo Y, Ribeiro JM, Anderson JM, Bour S. dCAS: a desktop application for cDNA sequence annotation. Bioinformatics. 2009;25(9):1195-6.

38. Kim D, Pertea G, Trapnell C, Pimentel H, Kelley R, Salzberg SL. TopHat2: accurate alignment of transcriptomes in the presence of insertions, deletions and gene fusions. Genome Biol. 2013;14(4):R36.

39. Trapnell C, Hendrickson DG, Sauvageau M, Goff L, Rinn JL, Pachter L. Differential analysis of gene regulation at transcript resolution with RNA-seq. Nat Biotechnol. 2013;31(1):46-53.

40. Heberle H, Meirelles GV, da Silva FR, Telles GP, Minghim R. Interactivenn: a web-based tool for the analysis of sets through Venn diagrams. BMC Bioinformatics. 2015;16:169.

41. Goff L, Trapnell C, Kelley D. cummeRbund: Analysis, exploration, manipulation, and visualization of Cufflinks high-throughput sequencing data. In: R package version 2.10.0; 2013.

42. R Core Team: R: A language and environment for statistical computing. Vienna: R Foundation for Statistical Computing; 2019. [https://www.Rproject.org].

43. Götz S, García-Gómez JM, Terol J, Williams TD, Nagaraj SH, Nueda MJ, Robles M, Talón M, Dopazo J, Conesa A. High-throughput functional annotation and data mining with the Blast $2 \mathrm{GO}$ suite. Nucleic Acids Res. 2008;36(10):3420-35.

44. Al-Shahrour F, Díaz-Uriarte R, Dopazo J. FatiGO: a web tool for finding significant associations of gene ontology terms with groups of genes. Bioinformatics. 2004;20(4):578-80.

45. Katoh K, Standley DM. MAFFT multiple sequence alignment software version 7: improvements in performance and usability. Mol Biol Evol. 2013; 30(4):772-80.

46. Tamura K, Stecher G, Peterson D, Filipski A, Kumar S. MEGA6: molecular evolutionary genetics analysis version 6.0. Mol Biol Evol. 2013;30(12):2725-9.

47. FigTree [http://tree.bio.ed.ac.uk/software/figtree].

48. Jones DT. Improving the accuracy of transmembrane protein topology prediction using evolutionary information. Bioinformatics. 2007;23(5):538-44.

49. Johns SJ. TOPO2, Transmembrane protein display software. [http://www. sacs.ucsf.edu/TOPO2/]. Accessed 5 May 2018.

50. Van der Auwera GA, Carneiro MO, Hartl C, Poplin R, Del Angel G, LevyMoonshine A, Jordan T, Shakir K, Roazen D, Thibault J, et al. From FastQ data to high confidence variant calls: the genome analysis Toolkit best practices pipeline. Curr Protoc Bioinformatics. 2013;43:11.10.11-33.

51. Picard [http://broadinstitute.github.io/picard]. Accessed 1 Mar 2014.

52. DePristo MA, Banks E, Poplin R, Garimella KV, Maguire JR, Hartl C, Philippakis AA, del Angel G, Rivas MA, Hanna M, et al. A framework for variation discovery and genotyping using next-generation DNA sequencing data. Nat Genet. 2011;43(5):491-8.

53. McDonald $\mathrm{JH}$, Kreitman M. Adaptive protein evolution at the Adh locus in Drosophila. Nature. 1991;351(6328):652-4.

54. Rozas J, Ferrer-Mata A, Sánchez-DelBarrio JC, Guirao-Rico S, Librado P, Ramos-Onsins SE, Sánchez-Gracia A. DnaSP 6: DNA sequence polymorphism analysis of large data sets. Mol Biol Evol. 2017;34(12):3299-302.

55. Thorvaldsdóttir H, Robinson JT, Mesirov JP. Integrative genomics viewer (IGV): high-performance genomics data visualization and exploration. Brief Bioinform. 2013;14(2):178-92.

56. Untergasser A, Cutcutache I, Koressaar T, Ye J, Faircloth BC, Remm M, Rozen SG. Primer3 - new capabilities and interfaces. Nucleic Acids Res. 2012;40(15):e115.

57. Vandesompele J, De Preter K, Pattyn F, Poppe B, Van Roy N, De Paepe A, Speleman F. Accurate normalization of real-time quantitative RT-PCR data by geometric averaging of multiple internal control genes. Genome Biol. 2002;3(7):RESEARCH0034

58. Grigoraki L, Pipini D, Labbé P, Chaskopoulou A, Weill M, Vontas J. Carboxylesterase gene amplifications associated with insecticide resistance in Aedes albopictus: geographical distribution and evolutionary origin. PLoS Negl Trop Dis. 2017;11(4):e0005533.

59. Poelchau MF, Reynolds JA, Denlinger DL, Elsik CG, Armbruster PA. A de novo transcriptome of the Asian tiger mosquito, Aedes albopictus, to identify candidate transcripts for diapause preparation. BMC Genomics. 2011;12:619.

60. Dritsou V, Topalis P, Windbichler N, Simoni A, Hall A, Lawson D, Hinsley M, Hughes D, Napolioni V, Crucianelli F, et al. A draft genome sequence of an invasive mosquito: an Italian Aedes albopictus. Pathog Glob Health. 2015; 109(5):207-20.
61. Chen XG, Jiang X, Gu J, Xu M, Wu Y, Deng Y, Zhang C, Bonizzoni M, Dermauw W, Vontas J, et al. Genome sequence of the Asian Tiger mosquito, Aedes albopictus, reveals insights into its biology, genetics, and evolution. Proc Natl Acad Sci U S A. 2015;112(44):E5907-15.

62. Manoharan M, Ng Fuk Chong M, Vaïtinadapoulé A, Frumence $E$, Sowdhamini R, Offmann B. Comparative genomics of odorant binding proteins in Anopheles gambiae, Aedes aegypti, and Culex quinquefasciatus. Genome Biol Evol. 2013;5(1):163-80.

63. Bohbot J, Pitts RJ, Kwon HW, Rützler M, Robertson HM, Zwiebel LJ. Molecular characterization of the Aedes aegypti odorant receptor gene family. Insect Mol Biol. 2007;16(5):525-37.

64. Bohbot JD, Sparks JT, Dickens JC. The maxillary palp of Aedes aegypti, a model of multisensory integration. Insect Biochem Mol Biol. 2014;48:29-39.

65. Lombardo F, Salvemini M, Fiorillo C, Nolan T, Zwiebel LJ, Ribeiro JM, Arcà B. Deciphering the olfactory repertoire of the tiger mosquito Aedes albopictus. BMC Genomics. 2017;18(1):770.

66. Hoshino K, Isawa H, Tsuda Y, Sawabe K, Kobayashi M. Isolation and characterization of a new insect flavivirus from Aedes albopictus and Aedes flavopictus mosquitoes in Japan. Virology. 2009;391(1):119-29.

67. Haddow AD, Guzman H, Popov VL, Wood TG, Widen SG, Tesh RB, Weaver SC. First isolation of Aedes flavivirus in the Western hemisphere and evidence of vertical transmission in the mosquito Aedes (Stegomyia) albopictus (Diptera: Culicidae). Virology. 2013;440(2):134-9.

68. Mair P, Wilcox R. Robust statistical methods in R using the WRS2 package. Behav Res Methods. 2020;52(2):464-88.

69. Xu P, Leal WS. Probing insect odorant receptors with their cognate ligands: insights into structural features. Biochem Biophys Res Commun. 2013;435(3): 477-82.

70. Hughes DT, Wang G, Zwiebel LI, Luetje CW. A determinant of odorant specificity is located at the extracellular loop 2-transmembrane domain 4 interface of an Anopheles gambiae odorant receptor subunit. Chem Senses. 2014;39(9):761-9.

71. Vosshall LB, Hansson BS. A unified nomenclature system for the insect olfactory coreceptor. Chem Senses. 2011;36(6):497-8.

72. DeGennaro M, McBride CS, Seeholzer L, Nakagawa T, Dennis EJ, Goldman C, Jasinskiene $\mathrm{N}$, James AA, Vosshall LB. orco mutant mosquitoes lose strong preference for humans and are not repelled by volatile DEET. Nature. 2013; 498(7455):487-91.

73. Mukunda L, Miazzi F, Kaltofen S, Hansson BS, Wicher D. Calmodulin modulates insect odorant receptor function. Cell Calcium. 2014;55(4):191-9.

74. Mukunda L, Miazzi F, Sargsyan V, Hansson BS, Wicher D. Calmodulin affects sensitization of Drosophila melanogaster odorant receptors. Front Cell Neurosci. 2016;10:28.

75. Karak S, Jacobs JS, Kittelmann M, Spalthoff C, Katana R, Sivan-Loukianova E, Schon MA, Kernan MJ, Eberl DF, Göpfert MC. Diverse roles of axonemal dyneins in Drosophila auditory neuron function and mechanical amplification in hearing. Sci Rep. 2015;5:17085.

76. Boo KS, Richards AG. Fine structure of scolopidia in Johnston's organ of female Aedes aegypti compared with that of the male. J Insect Physiol. 1975; 21(5):1129-39.

77. Nadrowski B, Effertz T, Senthilan PR, Göpfert MC. Antennal hearing in insects - new findings, new questions. Hear Res. 2011;273(1-2):7-13.

78. Armstrong JD, Texada MJ, Munjaal R, Baker DA, Beckingham KM. Gravitaxis in Drosophila melanogaster: a forward genetic screen. Genes Brain Behav. 2006:5(3):222-39.

79. Merrill CE, Riesgo-Escovar J, Pitts RJ, Kafatos FC, Carlson JR, Zwiebel LJ. Visual arrestins in olfactory pathways of Drosophila and the malaria vector mosquito Anopheles gambiae. Proc Natl Acad Sci U S A. 2002;99(3):1633-8.

80. Walker WB, Smith EM, Jan T, Zwiebel LJ. A functional role for Anopheles gambiae Arrestin1 in olfactory signal transduction. J Insect Physiol. 2008; 54(4):680-90.

81. Vogt RG, Miller NE, Litvack R, Fandino RA, Sparks J, Staples J, Friedman R, Dickens JC. The insect SNMP gene family. Insect Biochem Mol Biol. 2009; 39(7):448-56.

82. Jin $\mathrm{X}, \mathrm{Ha}$ TS, Smith DP. SNMP is a signaling component required for pheromone sensitivity in Drosophila. Proc Natl Acad Sci U S A. 2008;105(31): 10996-1001.

83. Pitts RJ, Rinker DC, Jones PL, Rokas A, Zwiebel LJ. Transcriptome profiling of chemosensory appendages in the malaria vector Anopheles gambiae reveals tissue- and sex-specific signatures of odor coding. BMC Genomics. 2011;12:271 
84. Matthews BJ, McBride CS, DeGennaro M, Despo O, Vosshall LB. The neurotranscriptome of the Aedes aegypti mosquito. BMC Genomics. 2016;17:32.

85. Sim S, Ramirez JL, Dimopoulos G. Dengue virus infection of the Aedes aegypti salivary gland and chemosensory apparatus induces genes that modulate infection and blood-feeding behavior. PLoS Pathog. 2012;8(3): e1002631.

86. Yuhua Deng, Hui Yan, Jinbao Gu, Jiabao Xu, Kun Wu, Zhijian Tu, Anthony A. James, Xiaoguang Chen, Wolfgang Blenau. Molecular and functional characterization of odorant-binding protein genes in an invasive vector mosquito, Aedes albopictus. PLoS ONE. 2013;8(7):e68836.

87. Tonya M. Colpitts, Jonathan Cox, Dana L. Vanlandingham, Fabiana M. Feitosa, Gong Cheng, Sebastian Kurscheid, Penghua Wang, Manoj N. Krishnan, Stephen Higgs, Erol Fikrig, Charles M. Rice. Alterations in the Aedes aegypti transcriptome during infection with West Nile, dengue and yellow fever viruses. PLoS Pathogens. 2011;7(9):e1002189.

88. Carolyn S. McBride, Felix Baier, Aman B. Omondi, Sarabeth A. Spitzer, Joel Lutomiah, Rosemary Sang, Rickard Ignell, Leslie B. Vosshall. Evolution of mosquito preference for humans linked to an odorant receptor. Nature. 2014;515(7526):222-27.

89. Erdelyan CNG, Mahood TH, Bader TSY, Whyard S. Functional validation of the carbon dioxide receptor genes in Aedes aegypti mosquitoes using RNA interference. Insect Molecular Biology. 2012;21(1):119-27.

90. Zhang D, Dimopoulos G, Wolf A, Miñana B, Kafatos FC, Winzerling JJ. Cloning and molecular characterization of two mosquito iron regulatory proteins. Insect Biochemist Mol Biol. 2002;32(5):579-89.

91. Zhou G, Velasquez L, Geiser DL, Mayo JJ, Winzerling JJ. Differential regulation of transferrin 1 and 2 in Aedes aegypti. Insect Biochemist Mol Biol. 2009;39(3):234-44.

92. Stéphane Tchankouo-Nguetcheu, Huot Khun, Laurence Pincet, Pascal Roux, Muriel Bahut, Michel Huerre, Catherine Guette, Valérie Choumet, Anthony R. Fooks. Differential protein modulation in midguts of Aedes aegypti infected with chikungunya and dengue 2 viruses. PLOS ONE. 2010;5(10):e13149.

93. Lefèvre T, Koella JC, Renaud F, Hurd H, Biron DG, Thomas F, Manchester M. New prospects for research on manipulation of insect vectors by pathogens. PLoS Pathogens. 2006;2(7):e72.

94. Ishak IH, Riveron JM, Ibrahim SS, Stott R, Longbottom J, Irving H, Wondji CS. The Cytochrome P450 gene CYP6P12 confers pyrethroid resistance in $k d r-$ free Malaysian populations of the dengue vector Aedes albopictus. Scientific Reports. 2016;6(1).

95. Vontas J, Kioulos E, Pavlidi N, Morou E, della Torre A, Ranson H. Insecticide resistance in the major dengue vectors Aedes albopictus and Aedes aegypti. Pesticide Biochemistry and Physiology. 2012;104(2):126-31.

96. Grigoraki L, Lagnel J, Kioulos I, Kampouraki A, Morou E, Labbé P, Weill M, Vontas J, McCall PJ. Transcriptome profiling and genetic study reveal amplified carboxylesterase genes implicated in temephos resistance, in the Asian tiger mosquito Aedes albopictus. PLoS Neglected Tropical Diseases. 2015;9(5):e0003771.

97. Scaraffia PY, Isoe J, Murillo A, Wells MA. Ammonia metabolism in Aedes aegypti. Insect Biochemistry and Molecular Biology. 2005;35(5):491-503.

98. Scaraffia PY, Zhang Q, Thorson K, Wysocki VH, Miesfeld RL. Differential ammonia metabolism in Aedes aegypti fat body and midgut tissues. Journal of Insect Physiology. 2010:56(9):1040-49.

99. Humberto Doriguêtto Gravina, Andreia Akemi Suzukawa, Camila Zanluca, Fatima María Cardozo Segovia, Marcel Kruchelski Tschá, Allan Martins da Silva, Helisson Faoro, Ricardo da Silva Ribeiro, Laura Patricia Mendoza Torres, Alejandra Rojas, Luis Ferrerira, Magda Clara Vieira da Costa Ribeiro, Adriana Delfraro, Claudia Nunes Duarte dos Santos. Identification of insect-specific flaviviruses in areas of Brazil and Paraguay experiencing endemic arbovirus transmission and the description of a novel flavivirus infecting Sabethes belisarioi. Virology. 2019;527:98-106.

100. Roiz D, Vázquez A, Rosso F, Arnoldi D, Girardi M, Cuevas L, Perez-Pastrana E, Sánchez-Seco MP, Tenorio A, Rizzoli A. Detection of a new insect flavivirus and isolation of Aedes flavivirus in Northern Italy. Parasites Vectors. 2012;5(1).

101. Roiz D, Ruiz S, Soriguer R, Figuerola J, Schneider BS. Landscape effects on the presence, abundance and diversity of mosquitoes in Mediterranean wetlands. PLOS ONE. 2015;10(6):e0128112.

102. Grisenti M, Vázquez A, Herrero L, Cuevas L, Perez-Pastrana E, Arnoldi D, Rosà R, Gioi C, Tenorio A, Sánchez-Seco MP, Rizzoli A. Wide detection of Aedes flavivirus in north-eastern Italy - a European hotspot of emerging mosquito-borne diseases. J Gen Virol. 2015;96(2):420-30.
103. Urbanelli S, Bellini R, Carrieri M, Sallicandro P, Celli G. Population structure of Aedes albopictus (Skuse): the mosquito which is colonizing Mediterranean countries. Heredity. 2000;84(3):331-37.

104. Goro K. Revisiting Houston and Memphis: The Background Histories Behind the discovery of the infestation by Aedes albopictus (Diptera: Culicidae) in the United States and their significance in the contemporary research. J Medi Entomol. 2012:49(6):1163-76.

105. Randolph VB, Hardy JL. Establishment and characterization of St Louis encephalitis virus persistent infections in Aedes and Culex mosquito cell lines. J Gen Virol. 1988;69(9):2189-98.

106. Newman CM, Krebs BT, Anderson TK, Hamer GL, Ruiz MO, Brawn JD, Brown WFH, Kitron UD, Goldberg TL. Culex flavivirus during West Nile virus epidemic and interepidemic years in Chicago, United States. Vector-Borne and Zoonotic Diseases. 2017;17(8):567-75.

107. Cohuet A, Krishnakumar S, Simard F, Morlais I, Koutsos A, Fontenille D, Mindrinos M, Kafatos FC. SNP discovery and molecular evolution in Anopheles gambiae, with special emphasis on innate immune system. BMC Genomics. 2008;9(1):227.

108. Morlais I, Severson DW. Intraspecific DNA variation in nuclear genes of the mosquito Aedes aegypti. Insect Mol Biol. 2003;12(6):631-39.

\section{Publisher's Note}

Springer Nature remains neutral with regard to jurisdictional claims in published maps and institutional affiliations.
Ready to submit your research? Choose BMC and benefit from:

- fast, convenient online submission

- thorough peer review by experienced researchers in your field

- rapid publication on acceptance

- support for research data, including large and complex data types

- gold Open Access which fosters wider collaboration and increased citations

- maximum visibility for your research: over $100 \mathrm{M}$ website views per year

At BMC, research is always in progress.

Learn more biomedcentral.com/submissions 